\title{
Current and future trends of additive manufacturing for chemistry applications: a review
}

\author{
Oyekunle Azeez Alimi ${ }^{1}$ and Reinout Meijboom ${ }^{1, *}$ (10 \\ ${ }^{1}$ Research Centre for Synthesis and Catalysis, Department of Chemical Sciences, University of Johannesburg, Auckland Park, \\ P.O. Box 524, Johannesburg 2006, South Africa
}

Received: 13 April 2021

Accepted: 17 July 2021

Published online:

13 August 2021

(C) The Author(s), under exclusive licence to Springer Science+Business Media, LLC, part of Springer Nature 2021

\begin{abstract}
Three-dimensional (3-D) printing, also known as additive manufacturing, refers to a method used to generate a physical object by joining materials in a layer-bylayer process from a three-dimensional virtual model. 3-D printing technology has been traditionally employed in rapid prototyping, engineering, and industrial design. More recently, new applications continue to emerge; this is because of its exceptional advantage and flexibility over the traditional manufacturing process. Unlike other conventional manufacturing methods, which are fundamentally subtractive, 3- $D$ printing is additive and, therefore, produces less waste. This review comprehensively summarises the application of additive manufacturing technologies in chemistry, chemical synthesis, and catalysis with particular attention to the production of general laboratory hardware, analytical facilities, reaction devices, and catalytically active substances. It also focuses on new and upcoming applications such as digital chemical synthesis, automation, and robotics in a synthetic environment. While discussing the contribution of this research area in the last decade, the current, future, and economic opportunities of additive manufacturing in chemical research and material development were fully covered.
\end{abstract}

Handling Editor: Maude Jimenez.

Address correspondence to E-mail: rmeijboom@uj.ac.za 


\section{GRAPHICAL ABSTRACT}

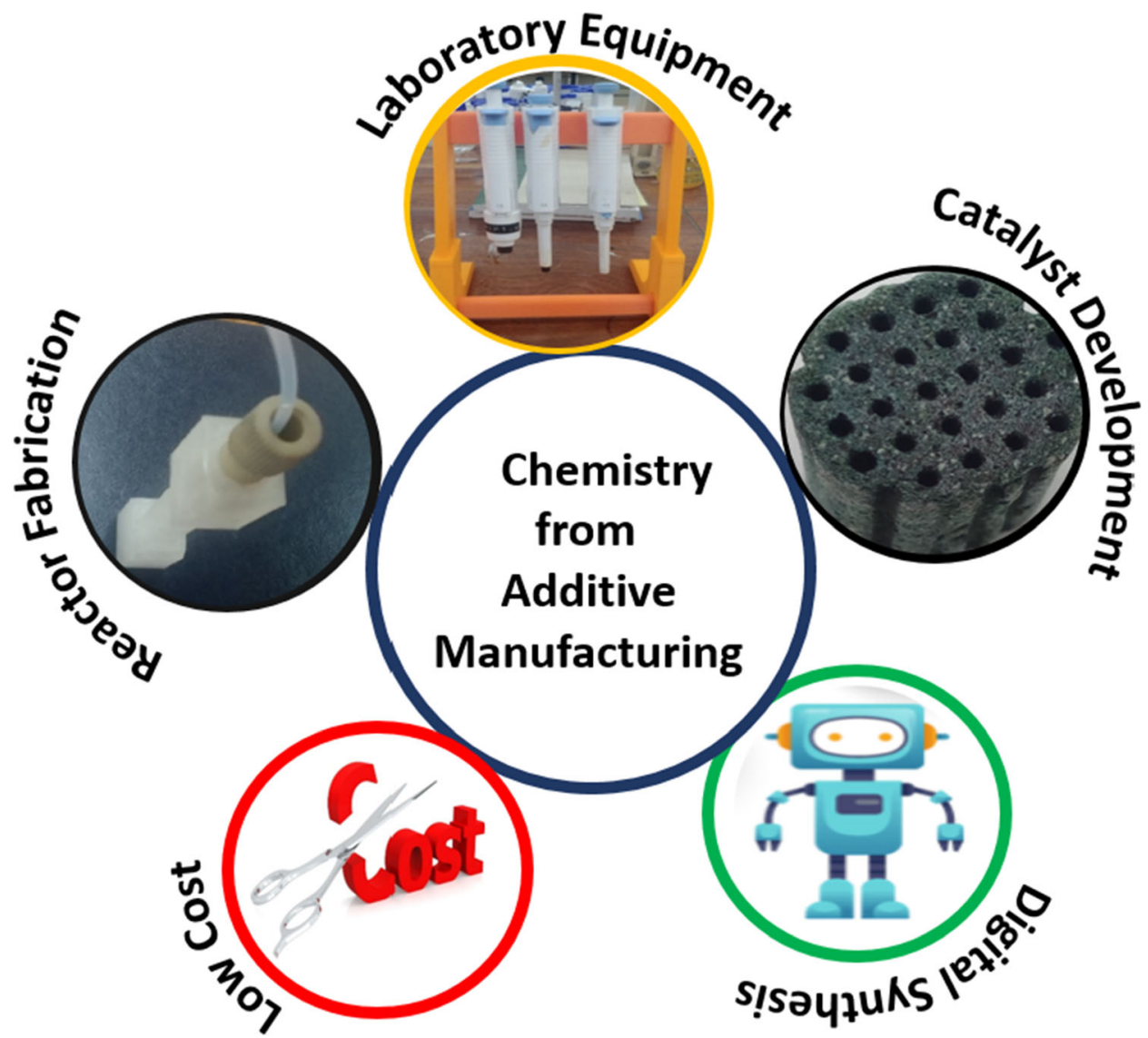

\section{Introduction}

The preparation of new materials and the synthesis of new products are currently key drivers for innovative and alternative technologies [1]. One of the leading technologies in this decade is $3-D$ printing [2]. Threedimensional $(3-D)$ printing, the most common form of additive manufacturing [3], is not new. It was invented in the mid-1980s [4] but was not utilised as a research tool until the early 2000s. However, with falling printer prices, affordability, and improved printer quality, 3-D printing has become an attractive choice for fabrication in chemistry, engineering, and associated fields [5]. Recently, 3-D printing has gained tremendous attention in the manufacturing and research arena due to its ability to create a physical object directly from a computer-aided design file (CAD), thus breaking traditional manufacturing protocols. Apart from these advantages, it is a cheap, fast, and precise method of device fabrication. These advantages have promoted its continuous relevance in the digital manufacturing of functioning devices and instruments.

\section{Three-dimensional printing technology}

Three-dimensional printing technology is categorised into different techniques; these include fused deposition modelling (FDM), also referred to as fused filament fabrication (FFF), inkjet printing, stereolithography, electron beam sintering, and selective layer sintering [6]. Among these printing technologies, fused deposition modelling (FDM) is 
the most popular and the cheapest. It involves the melting and solidification of a thermoplastic filament. In the typical procedure, a fresh thermoplastic filament is continuously fed into the heated chamber in the 3-D printer called the extruder. The molten polymer exits continuously in a threadlike manner through the nozzle. The nozzle is lowered to a levelled surface known as the print bed, depending on the desired resolution. As the molten filament gradually exits through the nozzle in a controlled manner, a pattern is created layer by layer on the print bed depending on the speed and the CAD design [7].

\section{Description of the additive manufacturing procedure}

The 3-D printing process is divided into two major steps, namely computer-aided design (modelling) and printing. As shown in Fig. 1, the first stage is the development of a computer-aided design (CAD). It may be created using a 3-D scanner, downloaded freely from open-sourced web-based $3-D$ printing communities (e.g. Tinkercard, Thingiverse, etc.), or appropriate $3-D$ creating software (e.g. Freecad, Tinkercad, Onshape, etc.) [8]. The model is usually exported as a stereolithographic file (STL), which is easily converted to a computer numerical control (Gcode) programming language to initiate printer control and movement using appropriate slicing software. The processed Gcode file is copied to the 3-D printer. The second stage is performed on the 3-D printer, which fabricates the object directly using layer-by-layer deposition of the molten filament on the print bed [9]. The schematic of the 3-D printing procedure is fully described in Fig. 1.

The flexibility of 3-D printing technology allows the fabrication of instruments, devices, and reactionwares of different properties for chemistry and chemical applications [11]. This is because of the availability of a different range of commercially available thermoplastic filaments such as polylactic acid (PLA) and acrylonitrile butadiene styrene (ABS) [12]. Moreover, with increasing awareness of 3-D printing technologies, other varieties of thermoplastic materials with suitable properties are now commercially available. This includes polypropylene (PP), polyetheretherketone (PEEK), poly (ethylene terephthalate) (PET), nylon, wood-PLA, and thermoplastic elastomer (TPE) [13]. Other printable materials, including ceramics, metals, and oxide of metals, have been reported.

Among different manufacturing methods, 3- $D$ printing is one of the cheapest, safest, most convenient, and energy-efficient [13]. The major advantage lies in the freedom of design, automation, and its additive nature, thus generating less waste. In chemistry and engineering, the development of the $3-D$ printing technique has contributed immensely to microfluidic device fabrication [14]. According to recent data available from the Scopus citation database, Fig. 2 reveals the trend in the relevance of 3- $D$ printing in microfluidic device fabrication in terms of research output (publications) from 2002 to 2020. It has made reactors more accessible to researchers in various fields and accelerates innovation in the field of microfluidics $[15,16]$. Based on these figures, it is evident that 3-D printing has contributed immensely to the development of microfluidics as shown in the research trend.

As shown in Fig. 2, 3- $D$ printing was initially employed for the creation of master moulds because of its high degree of precision and accuracy (2002-2011). However, from 2011 to 2013, there was an increase in the number of scientific publications due to its relevance for the direct fabrication of
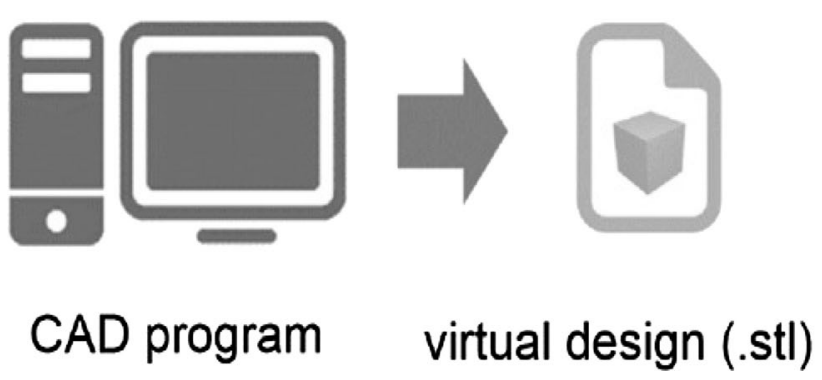

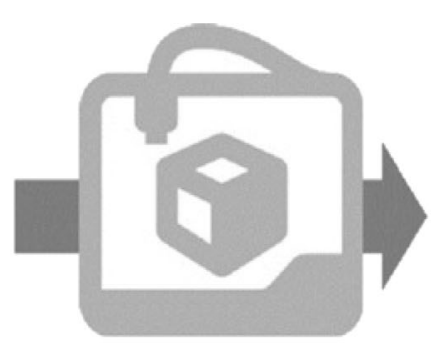

3D printer

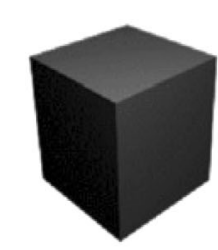

3D printed object

Figure 1 Schematic description of a 3-D printing procedure. Adapted with permission from reference [10]. Copyright [2017], [Wiley].

\section{Springer}




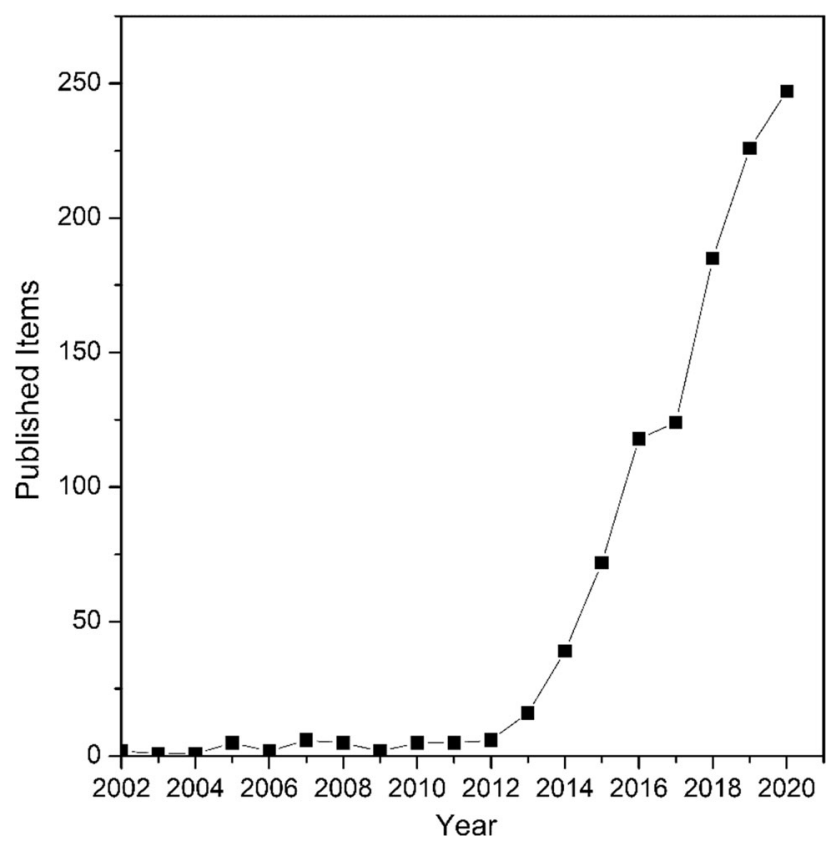

Figure 2 Published articles involving 3-D printing and microfluidic devices have surged from 2002 to 2020. The plot also reflects the most recent data searched (December 2020) from Scopus database. The following keywords were used: (microfluidics) and (3-D printing) from 2002 to 2020 .

microfluidic devices. Furthermore, from 2013 to 2015 an upsurge in the number of publications was reported. This is ascribed to the increasing demand for 3-D-printed reactionwares in other fields, especially in biological applications (Fig. 3) [8]. A similar trend was observed from 2015 to 2020, and this might be associated with the growing demand for $3-D$ printed devices in chemistry and other related fields.

\section{Role of 3-D printing in chemistry and associated fields}

"Why do I need a 3-D printer in my laboratory"? This is a likely response you will receive from a fellow researcher in chemistry when asking if they have a $3-D$ printer in their laboratory [17]. The 3-D printer is still perceived by most people as a device intended to create amusement, attention, trick, or a device for printing random tools. However, modern chemistry has proved that the 3-D printer has gone beyond an ordinary device for creating a gimmick. Its role has been extended to many scientific applications, including the fabrication of laboratory devices, electrochemical devices [18], microfluidic devices [19], and digital synthesis. Furthermore, it has also contributed significantly to the development of novel catalytic structures and materials such as graphene [20], monoliths, and metal-organic frameworks [21]. A significant advantage of this technique is the endless potential to fabricate devices or products that are not commercially available [22].

The past few decades have witnessed several technological innovations with significant impact in the world, and arguably, one of the best inventions is a 3-D printer [23]. The principle lies in a rapid and seamless translation of a computer model into a

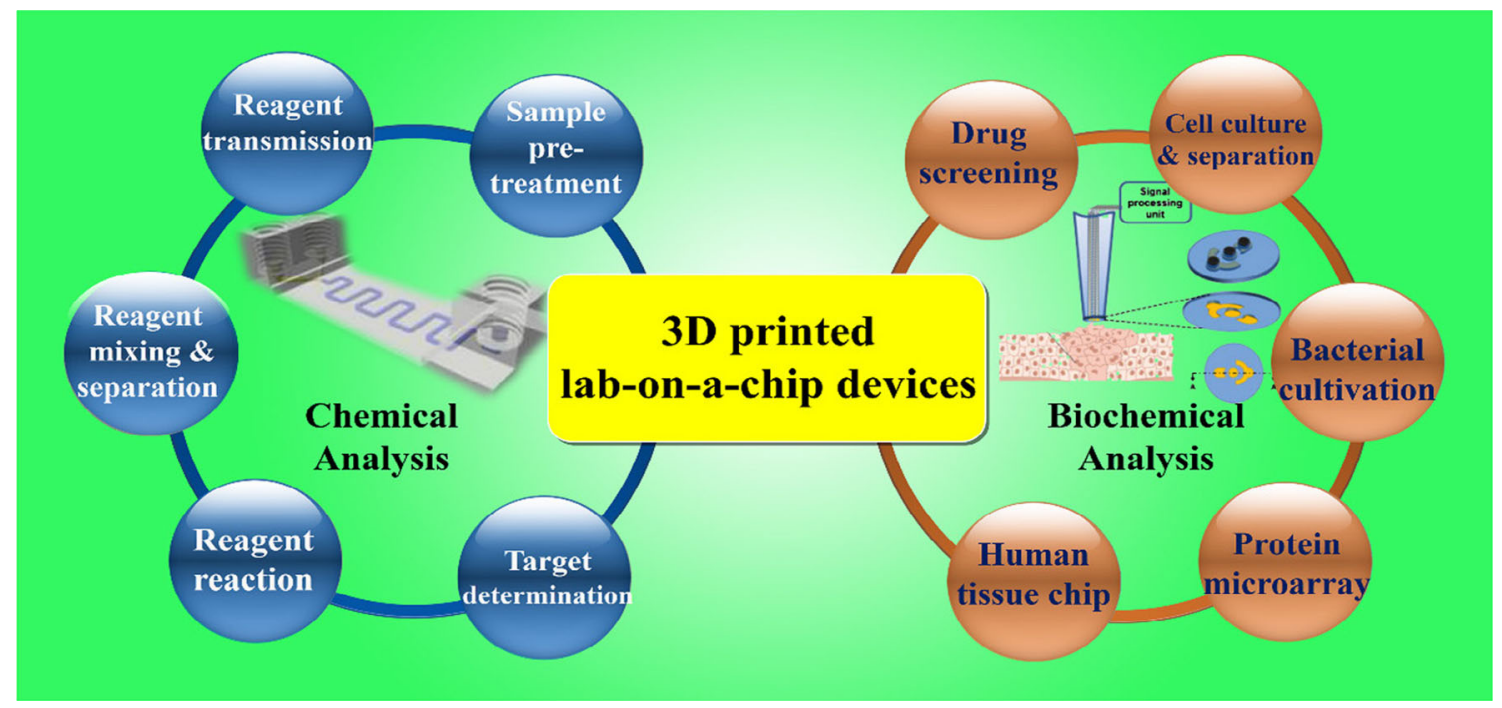

Figure 3 Application of $3-D$ printing in microfluidic devices fabrication in the chemical and biochemical analysis. Adapted with permission from reference [5]. Copyright [2016], [Elsevier]. 
physical object [24]. The unique potential of this technology is the ability to transform materials into functioning devices, which can be tailored for different experimental requirements.

\section{Laboratory hardware "on the go"}

One of the earliest applications of $3-D$ printing in chemistry is the production of laboratory equipment on demand. Most importantly, in the production of a range of open-source, functional laboratory hardware. This is highly beneficial from the current economic perspective, especially at this time when the world is facing a global pandemic challenge, which has disrupted the supply chain of essential goods and services. 3- $D$ printing technology has been utilised to address the manufacturing gaps created by COVID19 lockdowns by decentralising production. An extensive collection of computer-aided design (CAD) files is available online for free from open-source websites [25]. One of the earliest reports was published by Joshua Pearce [26]. He has reported the use of a 3-D printer to manufacture a range of laboratory devices, including a laboratory centrifuge [27], mixer and shaker [28], and a nutating mixer [29]. The pictures are presented in Fig. $4 a-c$. We have also achieved the design and fabrication of effective 3-Dprinted laboratory hardware, including a gas chromatography vial holder, a pipette stand, thermometer holder, etc. (Fig. 4d-f).

\section{Analytical instruments}

In addition to the standard laboratory hardware and tools, 3- $D$ printing has been employed for highly specialised analytical instruments. Cecil et al. [30] reported the fabrication of a LED-based capillary detector housing with an integrated slit, using a fused deposition modelling 3-D printer (Fig. 5a). The 3- $D$ printed photodetector compared favourably with the commercially available version. Similarly, Whitehead et al. [31] designed and fabricated a low-cost 3-Dprinted cuvette adapter for a traditional UV-visible spectrophotometer (Fig. 5b). The advantage of the adapter is that it can be used to interface different types of the cuvette to any traditional UV-visible spectrophotometer. The cuvette adapter also provides the opportunity to analyse liquid samples smaller than the required $2.5-3.5 \mathrm{ml}$ volume of the cuvette.
More recently, our research group has embarked on the digital manufacturing of a range of customised syringe pumps for catalytic applications. The pictures are presented in Fig. 5c, d.

\section{Teaching aids and model kits}

The application of 3-D printing technology has been extended further to the manufacturing of teaching aids. This is associated with the increasing number of accessible online open-source digital collections of printable educational models. It has not only lowered the price of instructional materials for teaching but has also enhanced the understanding of complicated scientific phenomena. In chemistry, 3-D-printed model kits have been employed in the teaching of orbitals, bonding, and molecular symmetry in the academic arena for undergraduate students [32-39]. Also, 3-D printing technology has provided an improved understanding of mesoporous materials using a tomographic imaging system [40].

More recently, we have started the manufacturing of model kits using our open-source 3-D printer for the teaching of undergraduate students in our university. The picture of the model kit is presented in Fig. 6e.

\section{Flow devices and reactors}

Catalysts, reactors, and flow devices are essential components of a catalytic system [41]. A catalyst can influence the reaction pathway, but the reactor plays a considerable role by providing an enabling environment to promote a catalytic function. Most research focuses on the preparation, characterisation, and evaluation of catalysts [42], while few reports are dedicated to catalysis and reactor development. Therefore, there is a need to conduct more research on the relationship between catalysis and reactor development to facilitate superior chemical synthesis.

Reactors (microreactors, millireactors, and mesoreactors) and microfluidic devices have been fabricated by injection moulding, laser ablation, lithography, and other traditional techniques [43]. However, to stay relevant with the demand for new and improved chemical synthesis, a new generation of chemical reactors must be developed [44]. This has propelled the search for new and improved fabrication methods for novel reactor development. Flow chemistry, microfluidics, and catalysis have benefited 

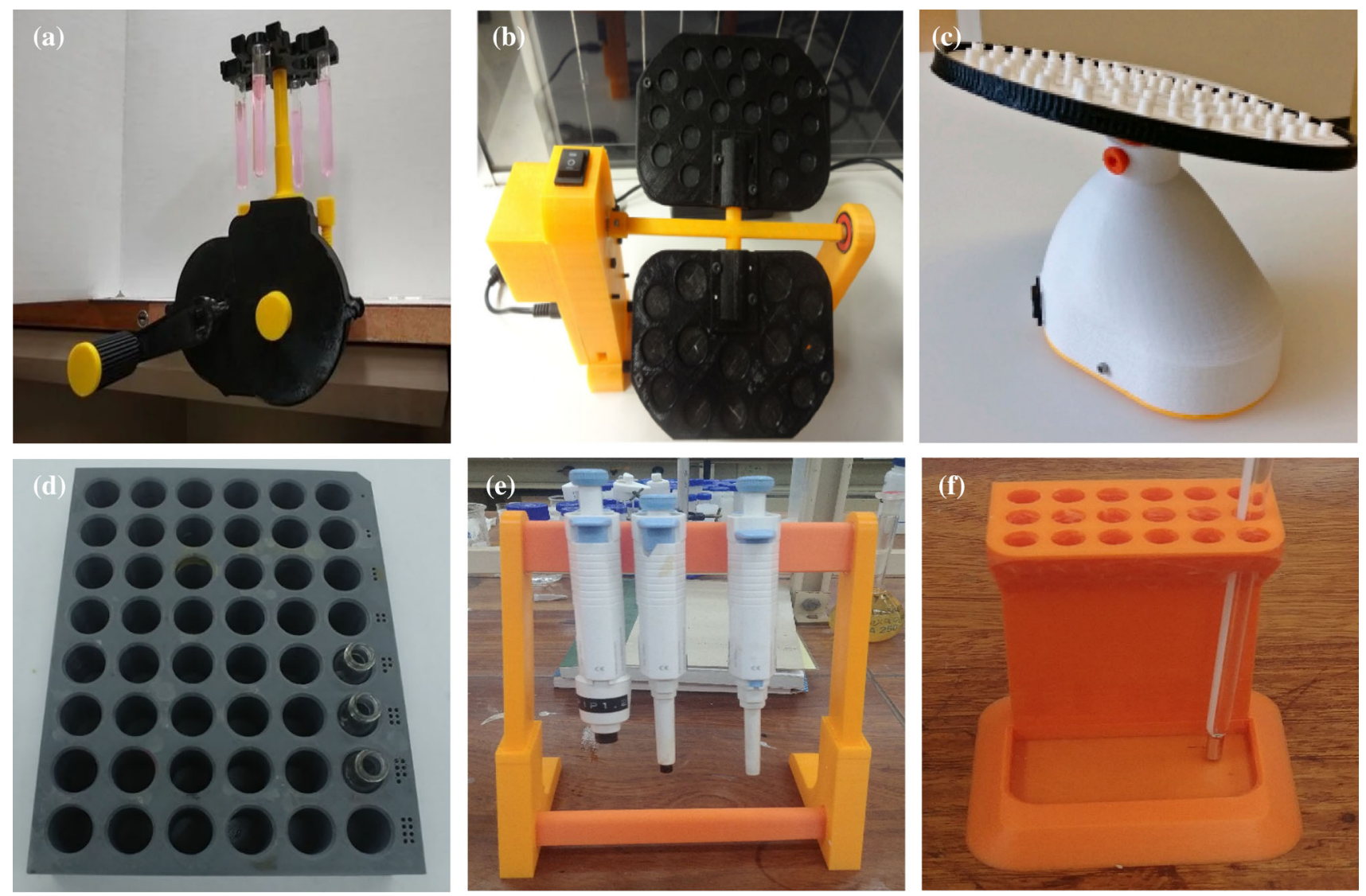

Figure 4 Pictures of 3-D-printed a laboratory centrifuge. Adapted with permission from reference [27]. Copyright [2019], [Multidisciplinary Digital Publishing Institute]. b Mixer and shaker. Adapted with permission from reference [28]. Copyright

from the available 3-D printing techniques such as direct ink writing (DIW) [45], fused deposition modelling (FDM) [22], stereolithography (SLA) [46], and selective layer sintering (SLS) [47] which are currently gaining attention in this research area because they are a simple, cheap, fast, and efficient manufacturing process. Apart from the economic advantages, another reason is the demand for flexible, customised, and tailor-made reaction devices for specific reaction set-ups.

This section presents an exemplary application of $3-D$ printing for the fabrication of reactor, reactor modules, and components for existing batch procedures, continuous-flow processes, and other specific applications. This is because additive manufacturing has been used to fabricate reaction devices and vessels with excellent heat transfer, good chemical compatibility, and impressive mechanical strength [48]. For instance, Wei et al. [41] reported the successful fabrication of iron, nickel, and cobalt metal
[2017], [Elsevier]. c Nutating mixer. Adapted with permission from reference [29]. Copyright [2017], [Multidisciplinary Digital Publishing Institute]. d GC vial holder, e pipette stand, f thermometer stand.

reactors by SLS 3-D printing. Unlike most reactors which require the use of catalytic materials, the reactor is self-catalysing because of the direct printing using $\mathrm{Fe}, \mathrm{Ni}$, and Co metals.

The 3-D-printed self-catalysing reactors are denoted as Fe-SCR, Ni-SCR, and Co-SCR. The reactors (FeSCR and Co-SCR) were employed for the preparation of liquid fuel from Fischer-Tropsch synthesis and $\mathrm{CO}_{2}$ hydrogenation, respectively. The nickel reactor (Ni-SCR) was applied for the production of syngas by $\mathrm{CO}_{2}$ reforming of methane. The image of the reactors is depicted in Fig. 7a. The catalytic activity of the printed Fe-SCR, Co-SCR, and Ni-SCR is presented in Fig. $7 \mathrm{~b}-\mathrm{e}$. In all the reactions tested, they obtained a very good conversion and excellent stability to the target product. In addition, they reported the excellent stability of the SCR catalytic system.

Anciaux et al. [49] reported the fabrication of an inexpensive microfluidic device using a consumergrade $3-D$ printer. The quality of the 3 - $D$-printed 

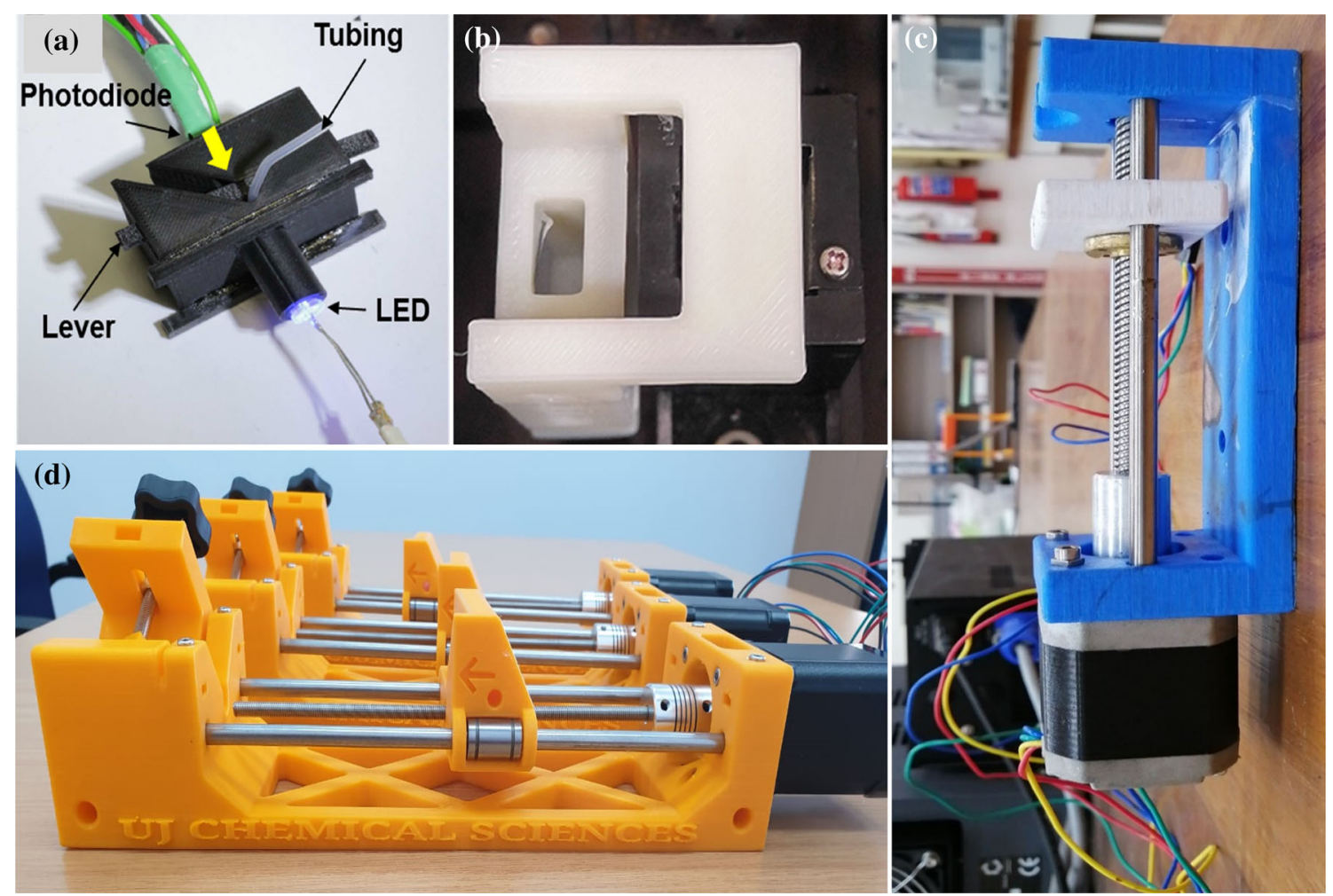

Figure 5 Pictures of 3-D-printed: a photodetector. Adapted with permission from reference [30]. Copyright [2017], [Elsevier]. b Universal adapter. Adapted with permission from reference [31].

reactor compared favourably with a similar device designed with glass material. Analysis of fluorescent dyes was performed in both 3-D-printed devices and glass fabricated device, and the result was comparable. Similarly, Rossi et al. [10] described the continuous-flow synthesis of enantiopure 1,2-amino alcohols via a palladium-catalysed Henry reaction in 3 - $D$-printed mesoreactors. In the experiment, reactors were fabricated with different filament materials (PLA, HIPS, nylon). Sizes, dimensions, and shapes were also varied, aimed at identifying the best performing reactor set-up. They reported the following average conversions 96\%, 97\%, and 92\% for PLA, nylon, and HIPS, respectively. Their target product's average enantioselectivity was obtained as $90 \%, 81 \%$, and $80 \%$, respectively.

The courtship between $3-D$ printing and continuous-flow processes has produced promising results that have opened up a large and unexploited avenue of possibilities in catalysis. Apart from these advantages and opportunities, $3-D$ printing has been highlighted as one of the essential components of the fourth industrial revolution. It also plays a significant
Copyright [2017], [American Chemical Society]. c Syringe pump (first generation). $\mathbf{d}$ Syringe pump (third generation).

role in economic competitiveness, allowing users to produce objects in accordance with their needs [50].

The use of 3-D printing has moved beyond the initial scope of manufacturing to the fabrication of functional devices. This is associated with the flexibility offered by additive manufacturing (AM) techniques to facilitate the rapid construction of complex fluidic architectures with a wide range of thermoplastic materials [51]. It has led to the fabrication of reactionware such as microfluidic and millifluidic devices with complicated internal geometry such as flow path, length, and volume for catalysis research and synthetic chemistry. The concept of reactionware was introduced by Leroy Cronin, who is one of the pioneer advocates in this research area [52]. Rao et al. reported the fabrication of a low-budget, chemically resistant 3-D-printed flow reactor for $S_{N} A r$ reactions [53]. The flow reactor was designed to interface with an existing flowSyn continuous-flow reactor system in order to minimise costs (Fig. 8) (Scheme 1).

They reported good chemical stability of the 3-Dprinted flow reactor in the presence of dimethylformamide, dichloroethane, and other solvents. They 

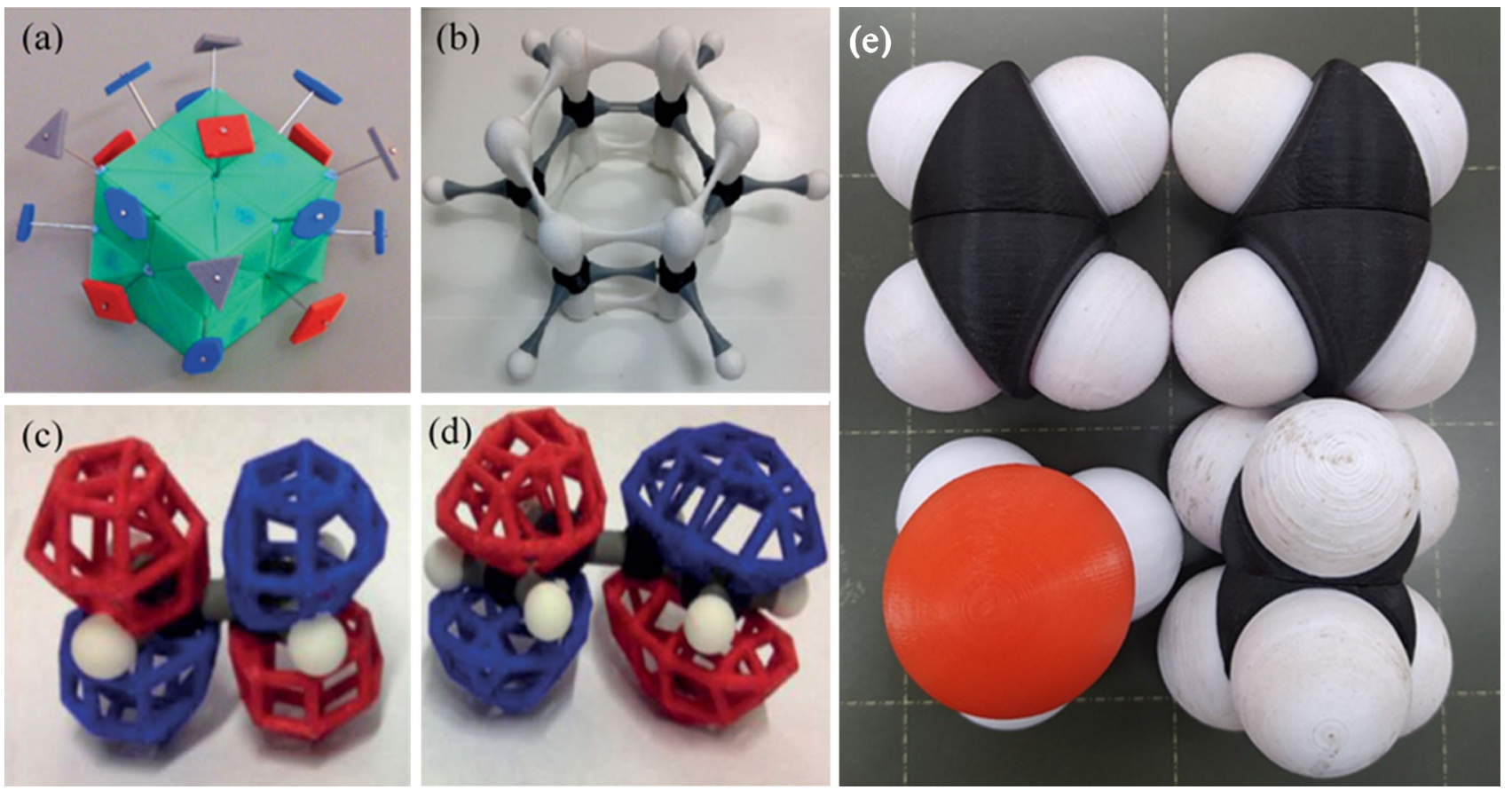

Figure 6 Pictures of 3-D-printed model kits. a 3-D-printed teaching aids for symmetry and point group identification. Adapted with permission from reference [39]. Copyright [2015], [American Chemical Society]. b 3-D-printed model of a benzene molecule. Adapted with permission from reference [33]. Copyright [2017], [American Chemical Society]. c Molecule of ethene showing the lowest unoccupied molecular orbital (LUMO). Adapted with

also reported excellent thermal stability of the printed reactor of up to $150{ }^{\circ} \mathrm{C}$ in the reaction. Also, the reactor demonstrated an excellent catalytic activity (66\% conversion of the substrate in $5 \mathrm{~min}$ of residence time). The reactor was reused five times without any loss in functionality and catalytic activity. Similarly, Neumaier et al. reported the synthesis of acetobromo- $\alpha$-D-glucose via glycosylation reactions (Scheme 2) in 3-D-printed flow reactors [54]. The reactor system and the flow set-up are presented in Fig. 9.

As shown in Scheme 2, they reported an excellent yield $(86 \%)$ of the product in 7.5 -min residence time.

In another study conducted by Dragone et al. [55], they performed the synthesis of imines (Scheme 3) and reduction of imines (Scheme 4) using a low-cost and chemically stable 3 - $D$-printed flow reactor. The reactor was integrated with an attenuated total reflectance-infrared (ATR-IR) flow cell to facilitate real-time monitoring and analysis during the reaction as shown in Fig. 10a. In the imine formation, they obtained a very high conversion of benzaldehyde permission from reference [38]. Copyright [2015], [American Chemical Society]. d Molecule of 1,3-butadiene showing the highest occupied molecular orbital (HOMO). Adapted with permission from reference [38]. Copyright [2015], [American Chemical Society]. e 3-D-printed model kits for the teaching of undergraduate students at the University of Johannesburg (unpublished).

(99\%) to imines in $7 \mathrm{~min}$ of residence time, while the reduction of imine was realised in $20 \mathrm{~s}$.

Additive manufacturing technology has been used to fabricate structured reactors with modified surfaces and geometry required for the desired catalytic processes [56]. Motivated by this ability, Kitson et al. [22] described the synthesis of phenanthridine-based heterocycles in a geometry-controlled 3- $D$-printed reaction device. The reactor consists of an inlet, outlet, and two catalyst compartments to promote different reaction steps, and the last compartment is laden with silica for the purification of the final product. Both catalysts were suspended in an acetoxy silicone adhesive material prior to the printing into the reactor via a robocasting method. The description of the catalytic system, fabrication procedure, and the catalytic evaluation are fully depicted in Fig. 11.

A major advantage of this device is the size; it is portable, is convenient, and combines multistep synthesis steps with purification [15]. Apart from the design and fabrication of flow devices and reactionwares, a plethora of modular 3-D-printed systems 
(a)

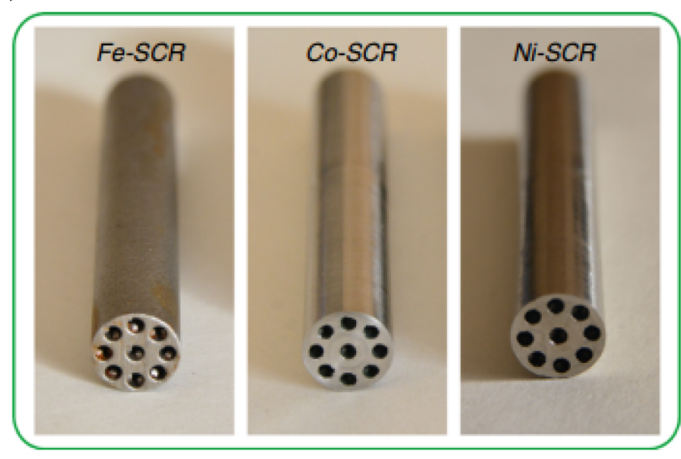

(c)

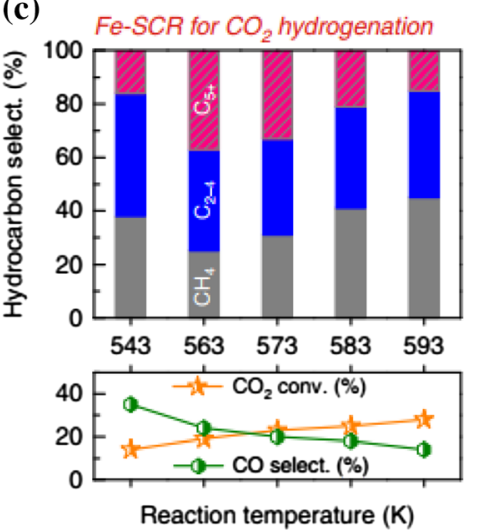

(b)

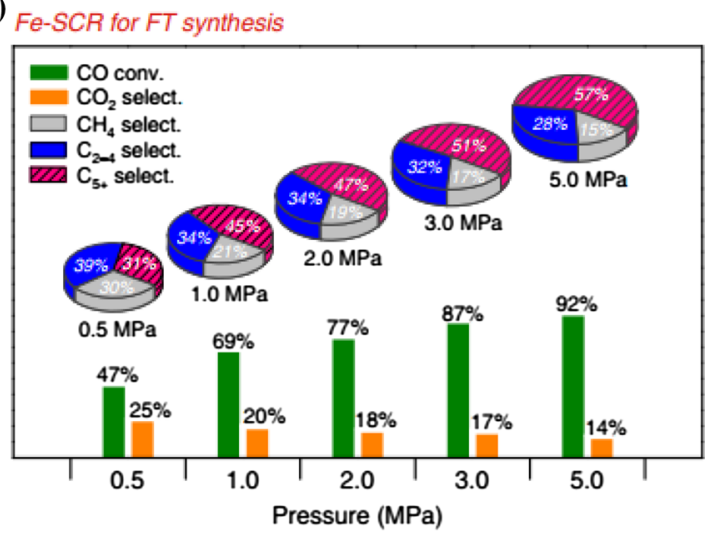

(e)

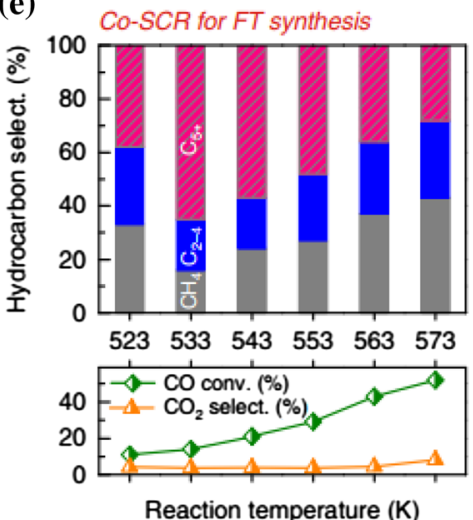

(e)

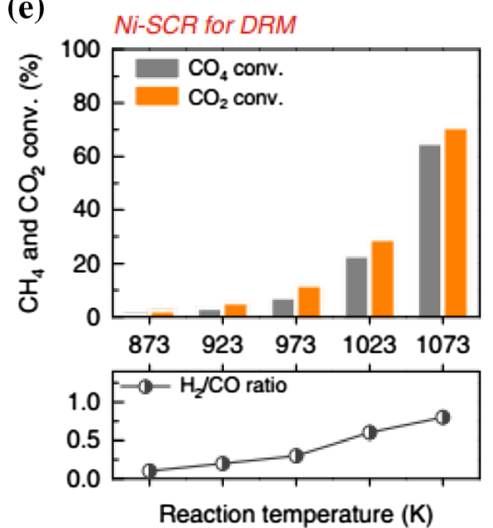

Figure 7 a Pictures of 3-D-printed self-catalysing reactors (FeSCR, Co-SCR, Ni-SCR), $\mathbf{b}$ catalytic activity of the Fe-SCR for FT synthesis, c catalytic activity of the Fe-SCR for $\mathrm{CO}_{2}$ hydrogenation, $\mathbf{d}$ catalytic activity of the Co-SCR for FT
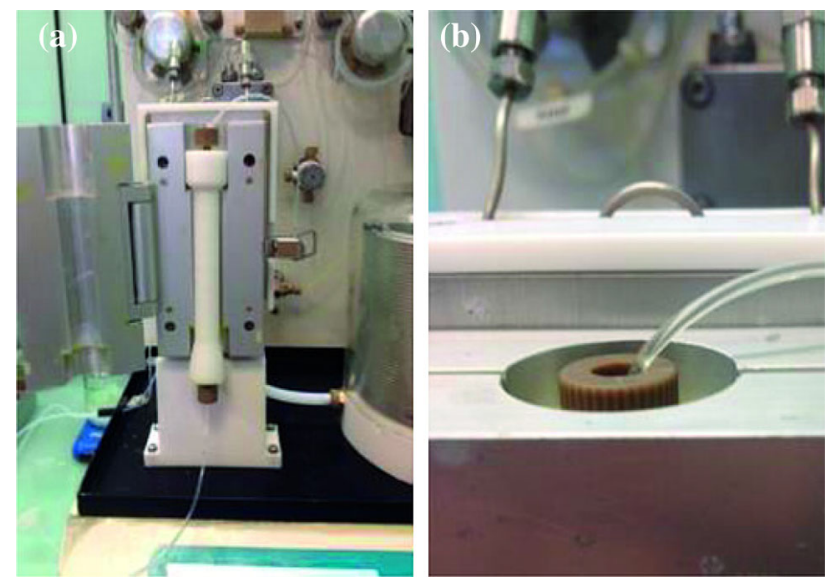

Figure 8 Picture of polypropylene-printed flow device and its integration into an existing flowSyn flow reaction system. Adapted with permission from reference [53]. Copyright [2017], [Wiley].

have been developed for chemical synthesis, which clearly shows the significance of $3-D$ printing towards a fully automated digital synthetic protocol. synthesis, e catalytic activity of the Ni-SCR for the reforming of methane. Adapted with permission from reference [41]. Copyright [2020], [Springer Nature].<smiles>O=[N+]([O-])c1ccccc1F</smiles>

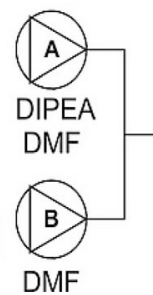<smiles>O=[N+]([O-])c1ccc(C2CC3CCC32)cc1NCCc1ccccc1</smiles>

Scheme $1 \mathrm{~S}_{\mathrm{N}} A$ r reactions between fluoronitrobenzene and amine promoted by $3-D$-printed flow reactor. Reprinted with permission from Rao et al. [53].

This idea emanated from the desire to design an automated synthetic procedure with little exposure and minimal human intervention [57]. Digitisation of chemistry also helps to address the difficulties in planning, setting up, and executing synthetic processes in the laboratory.

An excellent example is the digital synthesis of a muscle relaxant, baclofen, in a 3-D-printed flow reactor by Kitson et al. [57]. The reactionware consists 


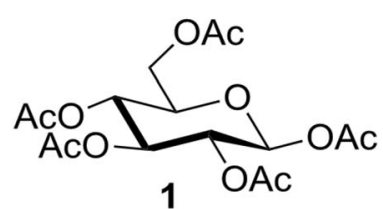

$1 \mathrm{M}$ solution in $\mathrm{CH}_{2} \mathrm{Cl}_{2}$ flow rate: $107 \mu \mathrm{L} / \mathrm{min}$

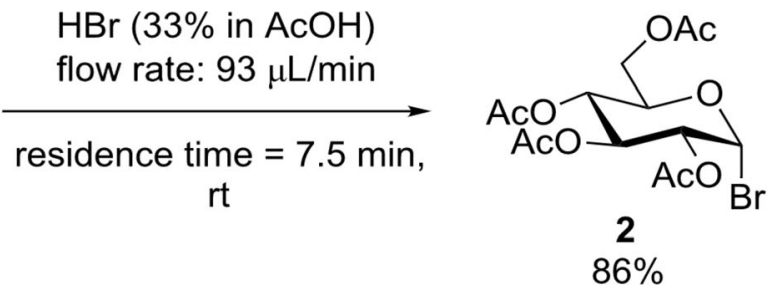

Scheme 2 Synthesis of acetobromo- $\alpha$-D-glucose 2 via glycosylation reaction. Adapted with permission from reference [54]. Copyright [2019], [Beilstein Institute for the Advancement of Chemical Sciences].

Figure 9 Schematic illustration of the flow set-up and the integrated 3-D-printed reactor system. Adapted with permission from reference [54]. Copyright [2019], [Beilstein Institute for the Advancement of Chemical Sciences].

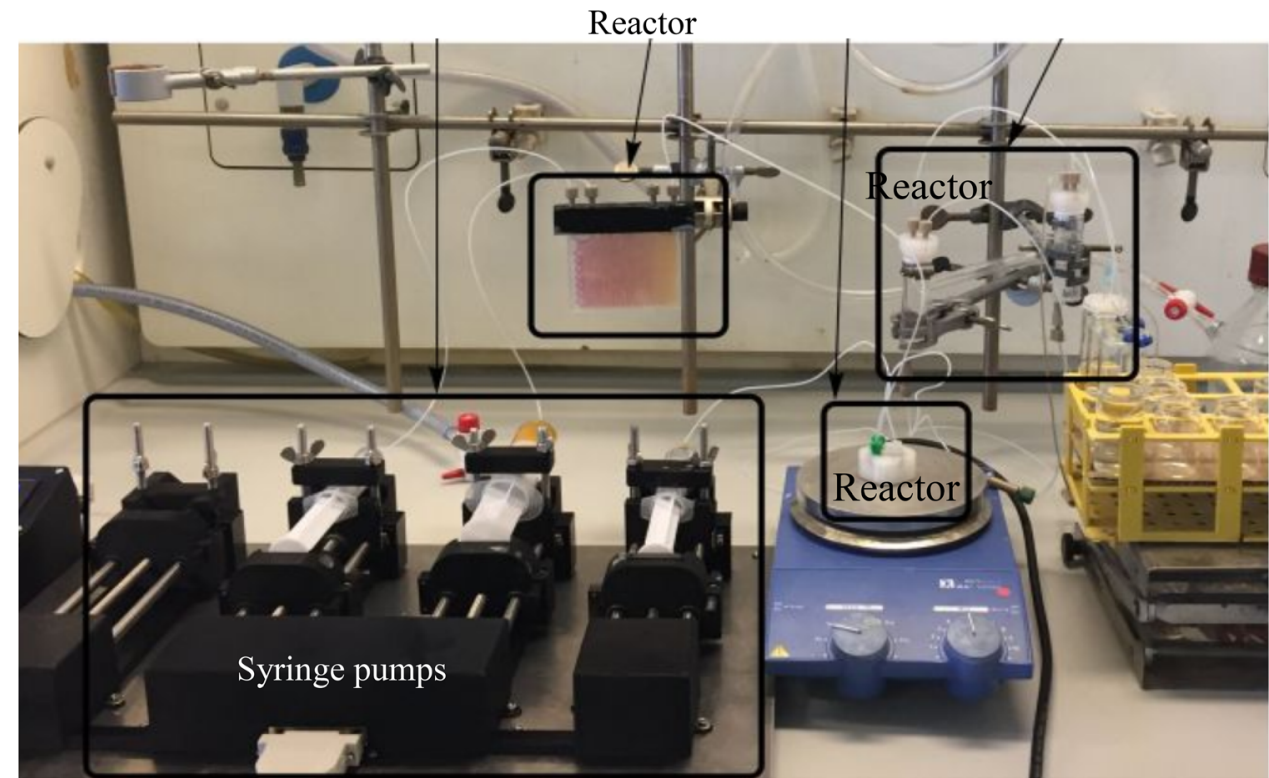<smiles>[R]c1cc([R])cc(/N=C/c2ccccc2)c1</smiles>

Scheme 3 Conversion of benzaldehyde to imines.

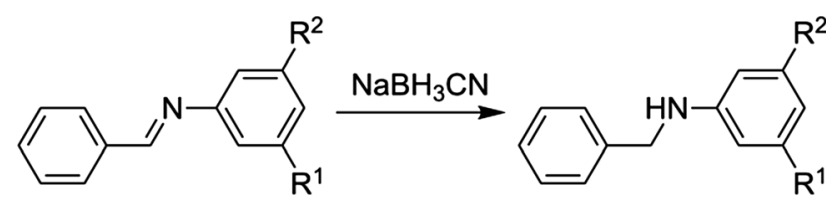

Scheme 4 Reduction of imine.

of an interconnected system of multiple cylindrical devices referred to as modules. The set-up is described in Fig. 12. Each of the modules is designed to perform a specific role in the synthetic process. As shown in Fig. 12, Michael addition, evaporation, and ether extraction are performed in module a, solvent extraction and reduction take place in module $b$, filtration and phase separation occur in c, hydrolysis and solvent exchange occur in module $d$, and the final stage where filtration occurs is in module e.

To justify the feasibility of their approach, the authors performed the same reactions in a convectional glass reactor, and the results are compared to the set-up in Scheme 5.

In 2016, the same group reported the digital synthesis of ibuprofen (an anti-inflammatory drug) using a modified open-source 3-D printer [58]. The printer was modified into a robot that can perform dual functions (3-D printing and liquid handling robot). The picture is depicted in Fig. 13a. The printer was initially used to fabricate a polypropylene-based reaction vessel for the preparation of ibuprofen, followed by dispensing of the starting materials into the printed vessel. In the procedure, the synthetic precursors (isobutylbenzene, propanoic acid, $\mathrm{PhI}(\mathrm{OAc})_{2} / \mathrm{TMOF}$, and $\left.\mathrm{KOH}\right)$ are preloaded into each of the syringe pumps which are independently controlled by a separate Arduino. The syringe pumps 
Figure 10 Picture of the a 3$D$-printed flow reactor integrated into the ATR-IR system and $\mathbf{b}$ CAD of the flow reactor. Adapted with permission from reference [55]. Copyright [2013], [Beilstein Institute for the Advancement of Chemical Sciences].
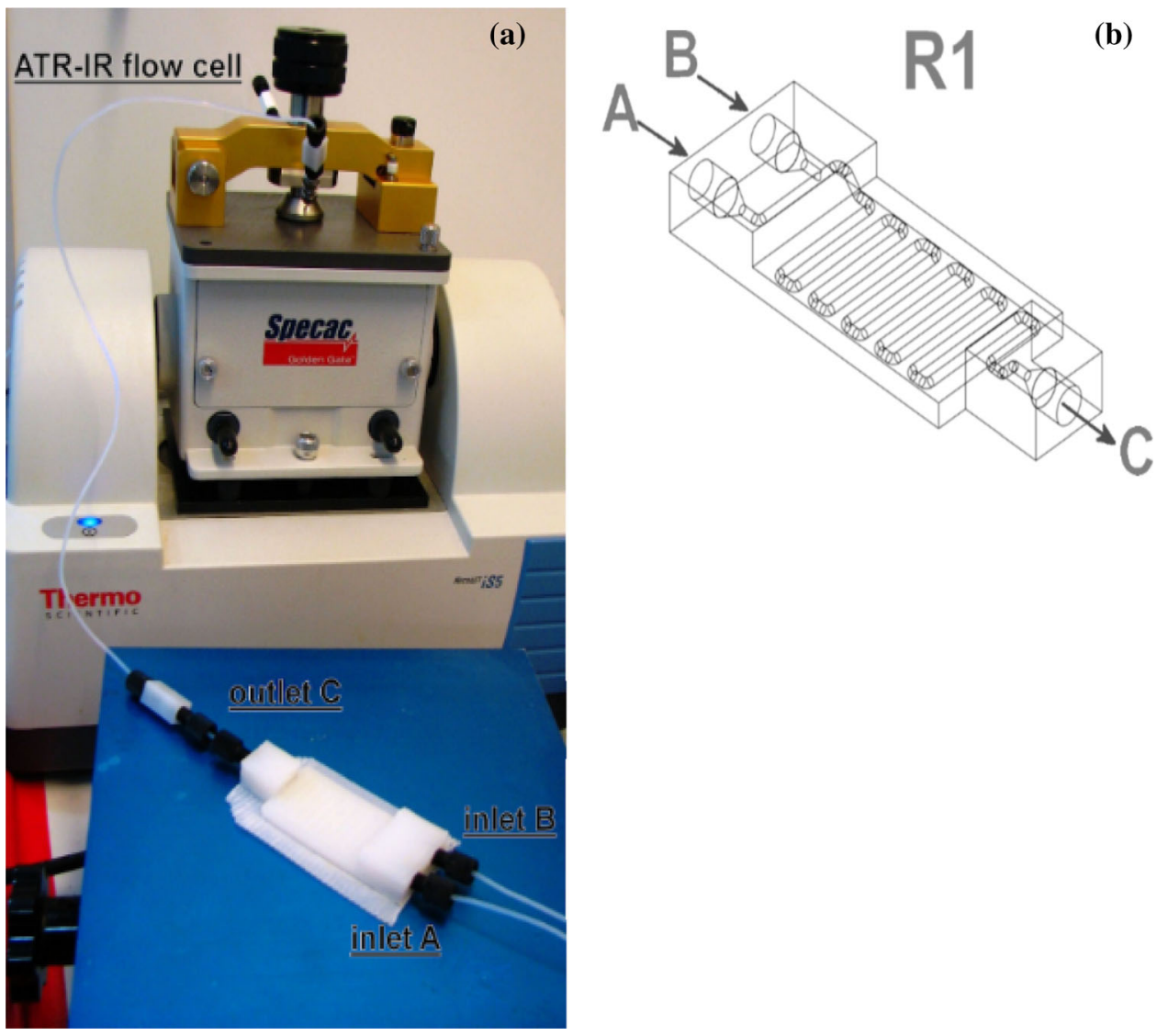

are connected to the extruder of the modified 3- $D$ printer via the Teflon tubing as shown in Fig. 13a. The syringe pumps are controlled to feed the printer with the starting materials, and in turn, the printer carefully dispenses the precursor into the printed reaction vessel at the required volume, rate, and order as shown in Fig. 13b.

Another major driver for the adoption of $3-D$ printing technology across scientific research is its flexibility. This technology offers great potential to incorporate additional features into new and existing facilities, either in batch and/or flow mode for improved functionalities. These capabilities led to the recent development of a "chemputer" by the Cronin group in 2018. The chemputer is a fully automatic system for doing chemistry which is controlled by the chemist [59]. The system is based on robotics and artificial intelligence approach. It is made up of interconnected series of modular reaction vessels with incorporated $3-D$-printed parts for additional functionalities. The combination of the modular setup is controlled by chemical code to facilitate a range of synthetic chemical transformations.
The modules consist of a traditional extraction, filtration, solvent exchange, and evaporator which are equipped with necessary gadgets for the execution of the specific task. However, it is digital and fully automated. Each of the modules is connected to a pump and valve to manipulate reactants and products. The chemputer is connected to a desktop computer which monitors and control the systems (pump, valves, etc.) with little human intervention. The picture of the chemputer is depicted in Fig. 14.

As a proof of concept, the group performed the synthesis of active pharmaceutical ingredients for three different drugs (viagra, rufinamide, and nytol) by just changing the software and chemical recipes [60]. They concluded that the chemputer has brought about a revolution in the synthesis of pharmaceutical ingredients. More recently, they published an updated version of the chemputer with additional modules and functionalities. The new synthesis machine can perform 8500 operations and process 17 different reactions using ten different modules [61].

Inspired by the studies in the literature, our research group has designed, fabricated, and 


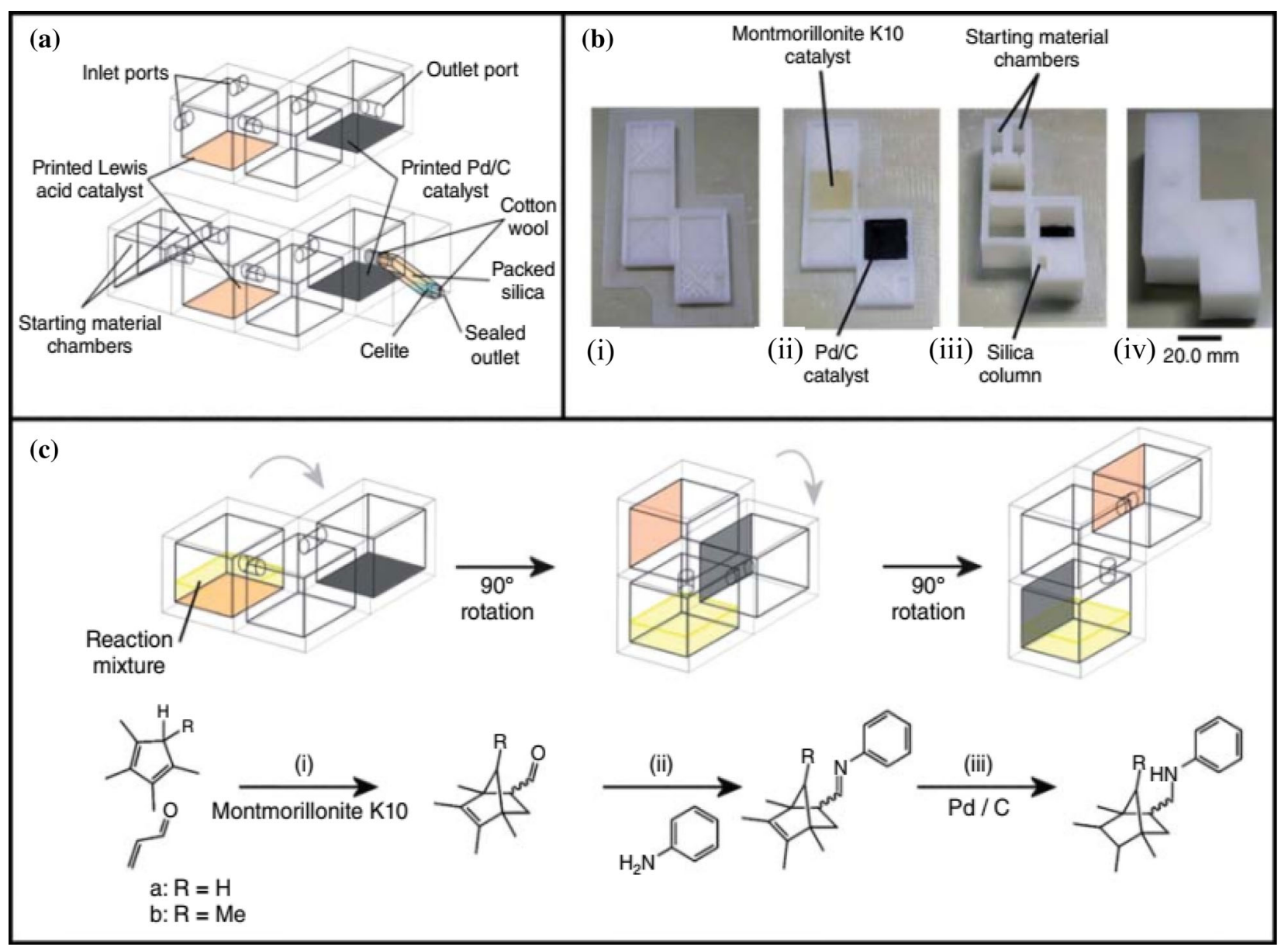

Figure 113 - $D$-printed geometry controlled catalytic device for multiple syntheses developed by the Cronin group. a Internal structure and design of the catalytic devices showing the different chambers, inlet and outlet, the position of the printed catalyst, and packed silica for purification of the final product. $\mathbf{b}$ Construction of the device. (1) The base of the reactor is printed in transparent PP filament using FDM 3-D printer. (2) The printed reactor base is transferred to a robocasting printer where the catalyst/acetoxy silicone paste was printed into the chambers. (3) The catalyst- imprinted device was transferred to the FDM printer for the printing to continue; starting material and silica were packed into the reactor at this stage. (4) Finally, the reactor was sealed and ready for use c operating procedure of the device, at each $90{ }^{\circ} \mathrm{C}$ rotation of the device, the substrates are transferred into the different catalyst compartment, and the corresponding products were formed. Adapted with permission from reference [22]. Copyright [2016], [Springer Nature].

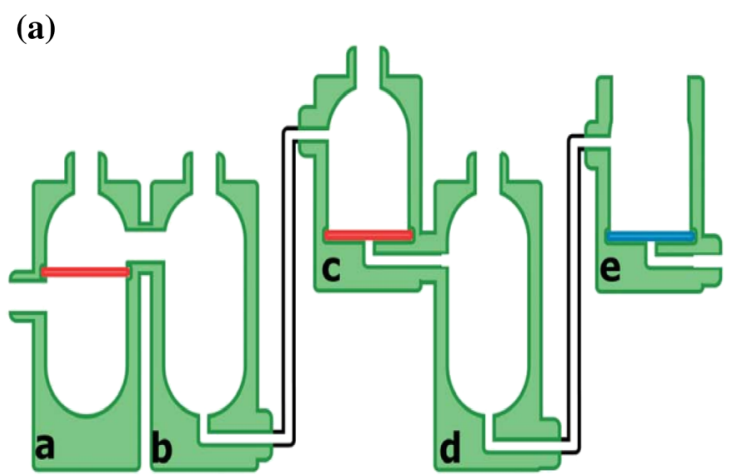

(b)

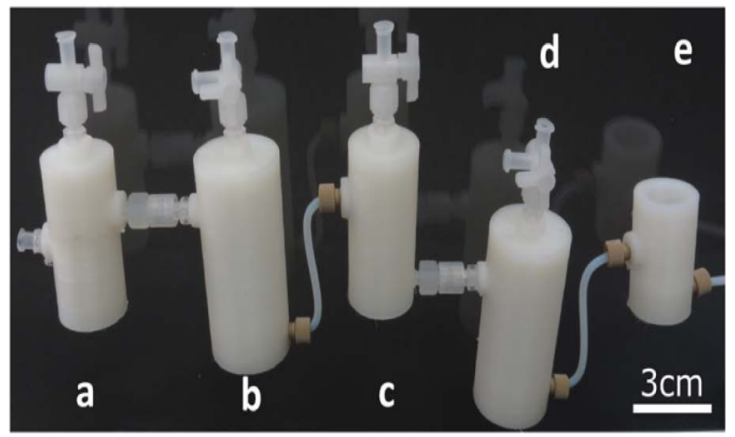

Figure 12 a Design of the modular 3-D-printed reactionware. b 3-D-printed modular reactionware. Adapted with permission from reference [57]. Copyright [2018], [American Association for the Advancement of Science]. 


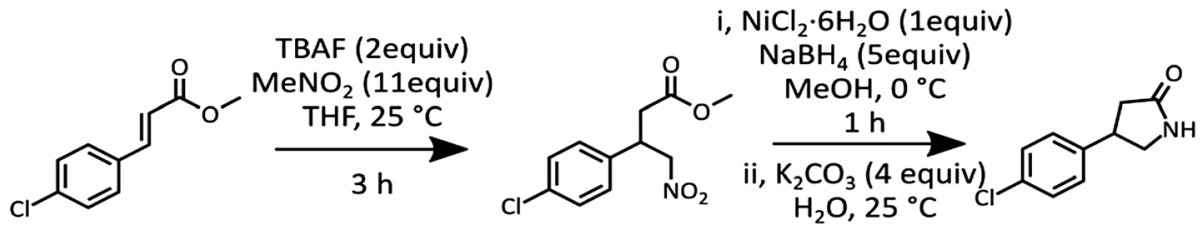

$2 \mathrm{~h}$

Isol. Yield: Glass (PP)
$59(47) \%$

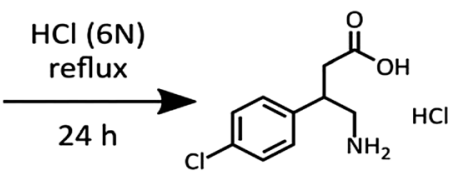

(士)-baclofen

Scheme 5 Comparative analysis of results (in terms of yield) between glass and polypropylene-printed modular reactor. Adapted with permission from reference [57]. Copyright [2018], [American Association for the Advancement of Science].
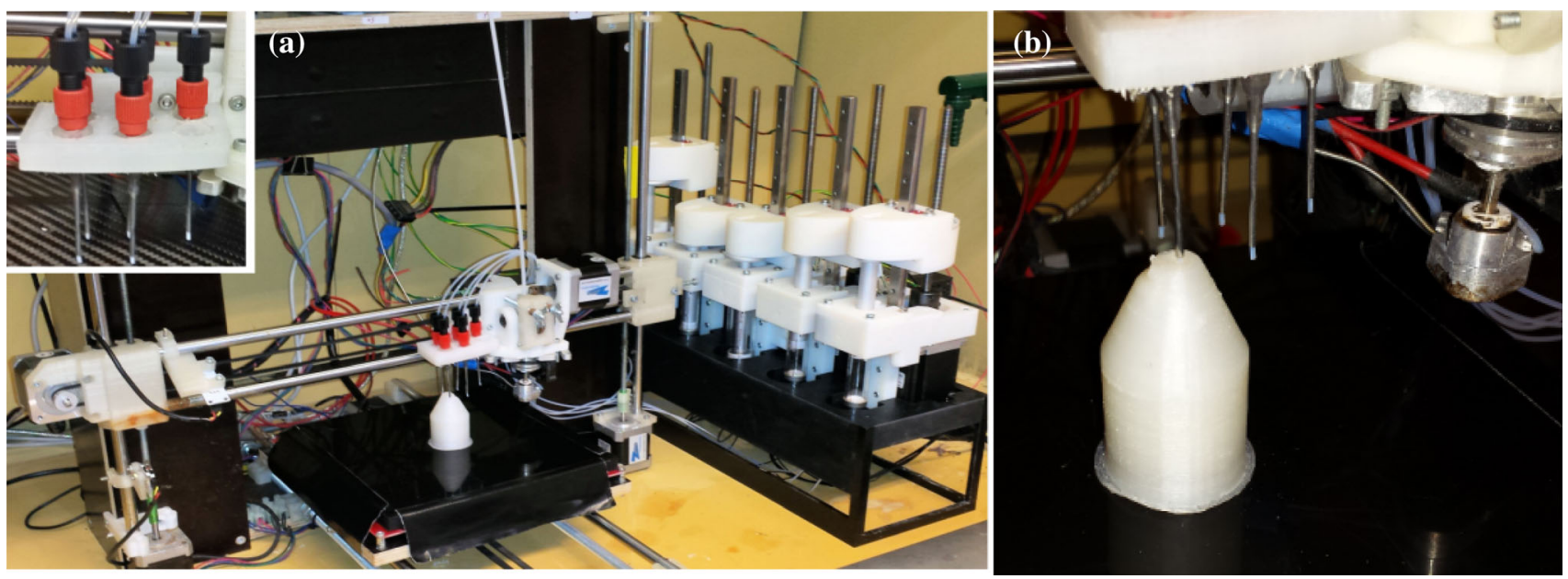

Figure 13 a 3-D-printed robot consisting of a modified opensource $3-D$ printer and inverted syringe pumps. b 3-D-printed reaction vessel and the Teflon-lined dispensing needle. Adapted

published a series of flow reactors to demonstrate the role of 3-D printing in catalysis research. The pictures are presented in Fig. 15. Full details of the design, fabrication, and catalytic evaluation are fully described in our recent publications [62-64]. 3-D printing has been employed to fabricate fittings and connectors for microfluidic connections [65].

Apart from chemical reactions and synthesis, 3- $D$ printed devices and objects have been deployed for water purification systems. In 2020, Zheng et al. [66] reported the application of a 3-D-printed photoreactor for water purification systems. The solar-powered 3-D-printed flow reactor was immobilised with $\mathrm{C}_{3} \mathrm{~N}_{4}$ supported on hydrogel beads to facilitate the water purification process. The design of the reactor is presented in Fig. 16. They reported that the reactor performance was comparable to the conventional slurry reactor for wastewater treatment. In addition, it also offers a convenient set-up for household water treatment. with permission from reference [58]. Copyright [2016], [Beilstein Institute for the Advancement of Chemical Sciences].

In a similar study conducted by Su et al. [67], they reported the application of a 3-D-printed device for the selective extraction/preconcentrator of trace elements in water samples. This is very important for the analytical detection of heavy metals in water. As shown in Fig. 17, the device consists of a reactor and an imprinted polyacrylate polymer monolith which helps to extract and concentrate the trace element in a flowing stream of water. The CAD design of the device is depicted in Fig. 17a, the 3-D-printed device is presented in Fig. $17 \mathrm{~b}$, and the $3-D$-printed polyacrylate monolithic extraction cuboid is presented in Fig. 17c. They reported that the device was highly efficient for the determination of heavy metals $(\mathrm{Mn}$, $\mathrm{Ni}, \mathrm{Zn}, \mathrm{Cu}, \mathrm{Cd}$, and $\mathrm{Pb}$ ) in seawater. The detection level of the device is also comparable with the conventional methods.

While most researchers reported excellent results and stability of the 3-D-printed reactors in batch and continuous-flow applications, it is worth mentioning 


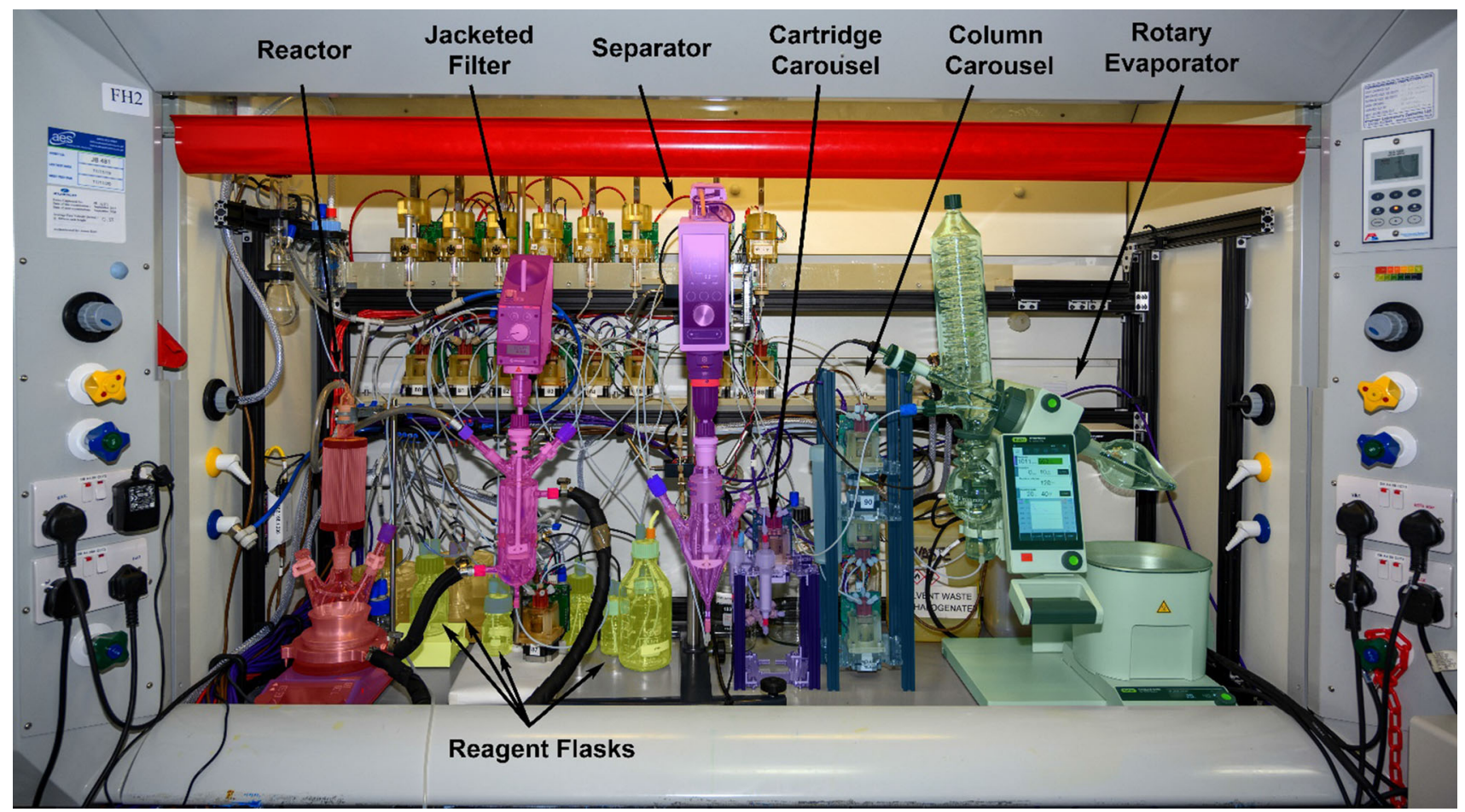

Figure 14 The chemputer (artificial chemist controlled by software). Adapted with permission from reference [59]. Copyright [2020], [Elsevier].

that it suffers from certain limitations such as poor chemical and thermal stability when compared to a regular metal reactor [68]. However, with improvement in the FDM techniques, printer quality, and commercial availability of more printing materials, flow devices with strong physical and chemical properties have been manufactured by $3-D$ printing. Currently, one of the best printing materials is polyetheretherketone (PEEK). PEEK is a mechanically strong, chemically stable, and temperature-resistant material. Because of these qualities, they are often used as fabrication of replacement parts for metals in a wide variety of applications. However, it is often a challenge to fabricate PEEK reaction devices by $3-D$ printing because of its extremely high melting temperature, making it difficult to extrude by FDM [69].

The first reported fabrication of PEEK using the FDM printing technique was published in 2013 by Valentan et al. [70]. In 2017, Yang et al. [71] also reported a successful extrusion of PEEK thermoplastic material using a modified $3-D$ printer. Both studies conducted by Valentan et al. [70] and Yang et al. [71] were not devoted to the development of chemical reactors or chemical synthesis. However, they are able to successfully demonstrate the possibility of 3-D printing with PEEK materials. More recently, Harding et al. [48] reported the fabrication of thermally stable and chemically resistant continuous-flow reaction devices with an integrated mixing compartment using PEEK thermoplastic filament with a modified FDM 3-D printer. The images of the CAD file and the 3-D-printed reactor are depicted in Fig. 18a, b.

To demonstrate the functionality of the printed PEEK flow device for chemical synthesis, the $S_{N} \mathrm{Ar}$ reaction of morpholine with 2,4-difluoronitrobenzene was performed in continuous flow. The reaction was carried out at $80{ }^{\circ} \mathrm{C}$ temperature, 100-psi pressure, and methanol as the solvent. The reaction scheme and the set-up are presented in Fig. 18c. They reported a very good chemical and thermal stability of the flow reactor. They also reported that the reactor could withstand an operating pressure of about 500 psi. In addition to the good synthetic functionality of the printed reactor, it also demonstrates an impressive liquid-liquid extraction functionality (more than 95\%).

In 2020, Menzel et al. [72] also demonstrated the possibility of fabrication of a complete reaction set-up 

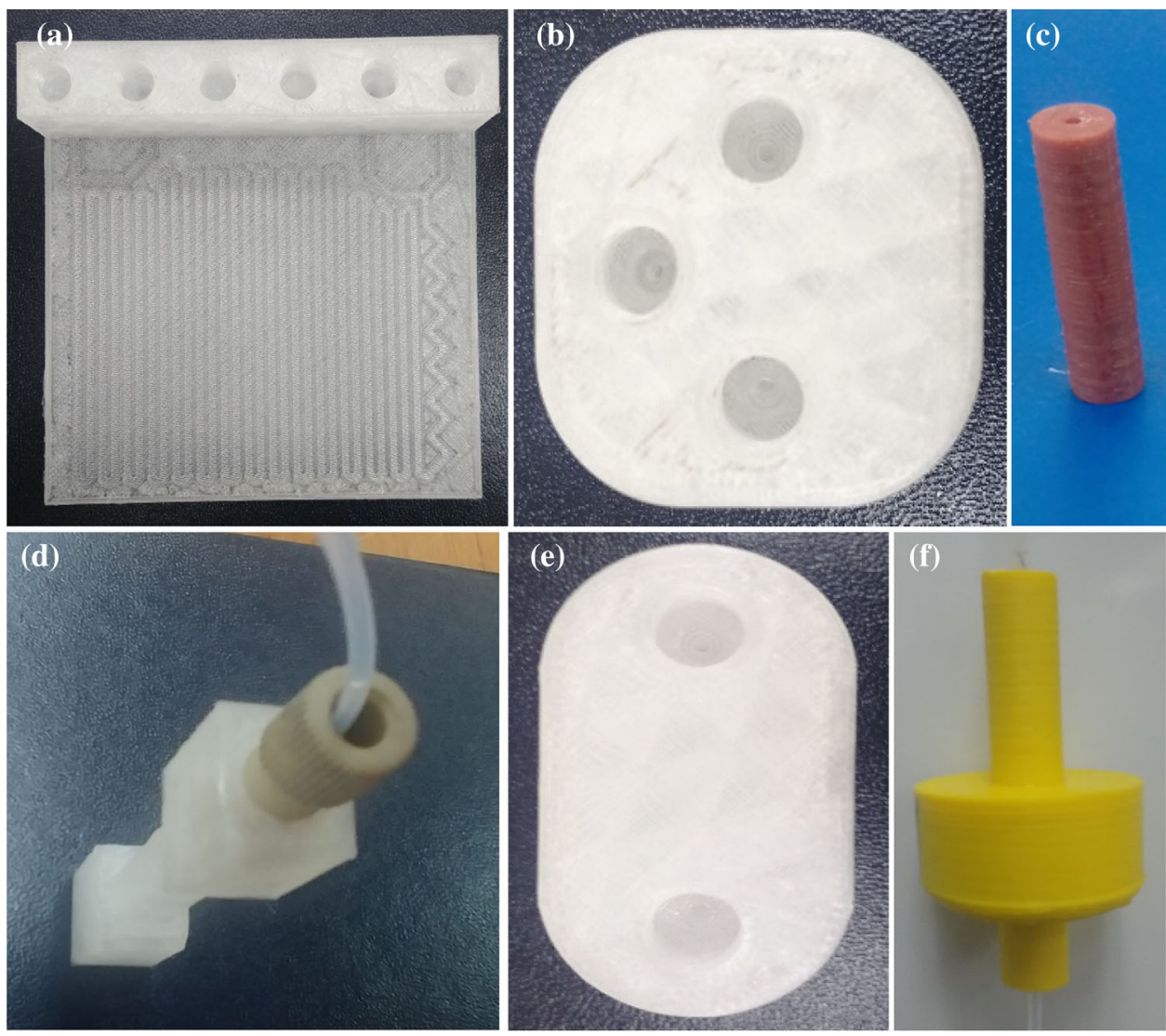

Figure 15 Pictures of 3-D-printed flow devices. a Adapted with permission from reference [54]. Copyright [2019], [Beilstein Institute for the Advancement of Chemical Sciences]. $\mathbf{b}$ Adapted with permission from reference [54]. Copyright [2019], [Beilstein Institute for the Advancement of Chemical Sciences]. c Adapted with permission from reference [62]. Copyright [2019], [Elsevier].

that is chemically resistant using PEEK thermoplastic materials with a modified $3-D$ printer. These include reactor, mixer, separator, backpressure regulator, and syringe pumps, which are capable of performing reactions at high temperatures. The pictures of the devices are presented in Fig. 19.

The performance of the printed reaction devices was evaluated for a range of multistep chemical reactions, including the conversion of ribose derivatives to a 2-fluoro-arabinose derivative. They reported an impressive conversion (90\%) with the reaction devices coupled with good stability, even at high temperatures.

3- $D$ printing is constantly evolving; the application of 3-D-printed devices has been extended to the synthesis of nanoparticles. Recently, Singh et al. [73] reported the synthesis of $\mathrm{Fe}_{3} \mathrm{O}_{4}, \mathrm{Ag}$, and $\mathrm{Au}$ nanoparticles using a droplet generator fabricated by d Adapted with permission from reference [63]. Copyright [2020], [Springer]. e Adapted with permission from reference [54]. Copyright [2019], [Beilstein Institute for the Advancement of Chemical Sciences]. f Adapted with permission from reference [64]. Copyright [2020], [Elsevier].

3-D printing. The schematic of the nanoparticle synthesis with the device is described in Fig. 20.

The prepared nanoparticles were characterised by UV and TEM to reveal the properties of the synthesised nanoparticles. They are also comparable to nanoparticles that are prepared using conventional methods.

\section{3-D printing of active catalytic material}

Another growing application of $3-D$ printing is its application in the construction of composite materials and structures depending on the feedstock directly used [74]. This is very beneficial for the preparation of functionalised 3-D catalytic structures in heterogeneous catalysis [75]. Unlike the traditional catalyst immobilisation method, immobilised three-dimensional-printed catalyst structures offer distinct 

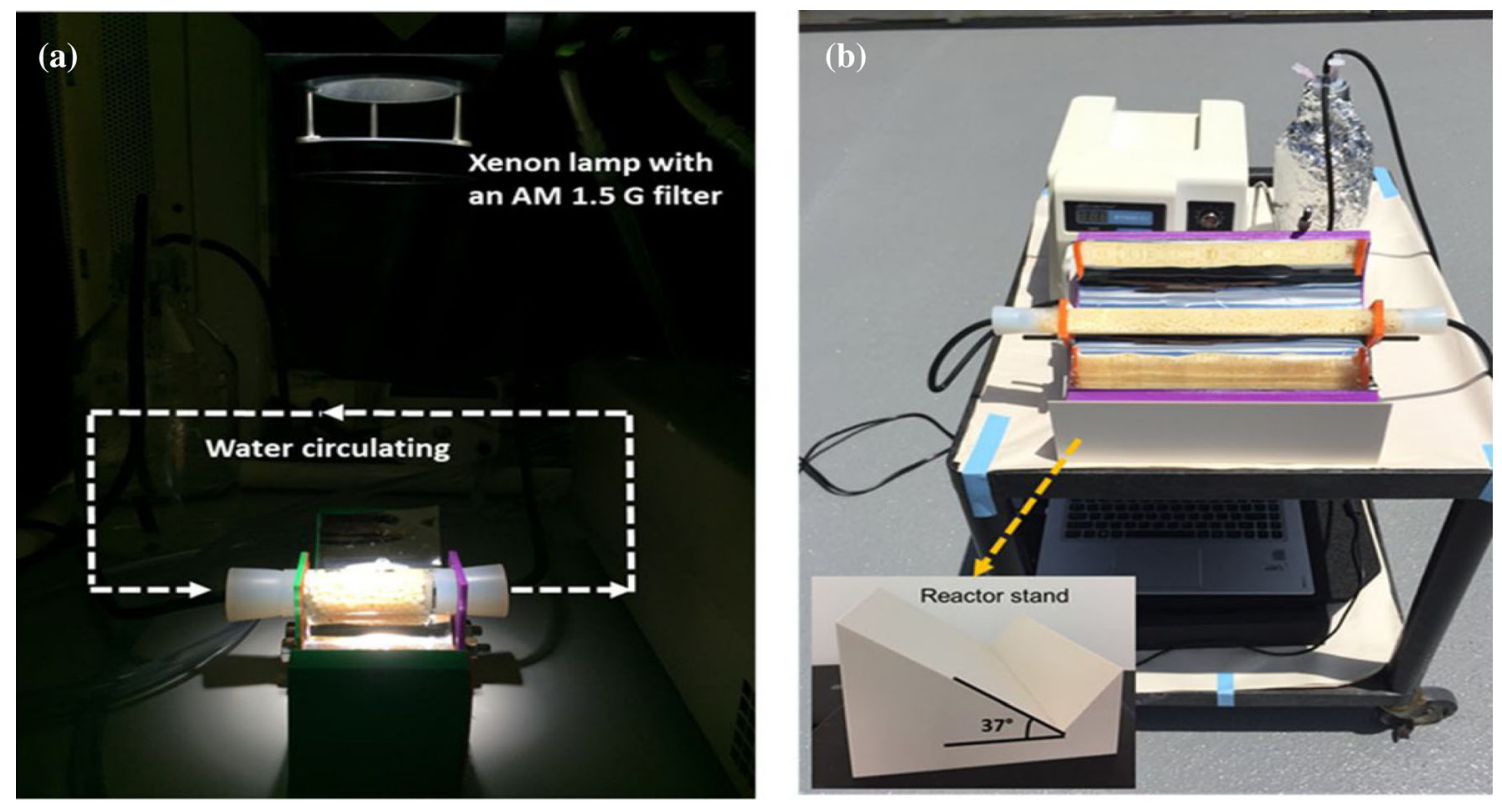

Figure 16 Pictures of 3-D-printed water purification system: (a) working principle of the reactor and (b) the reactor set-up and the reactor stand. Adapted with permission from reference [66]. Copyright [2020], [Elsevier].

(a)

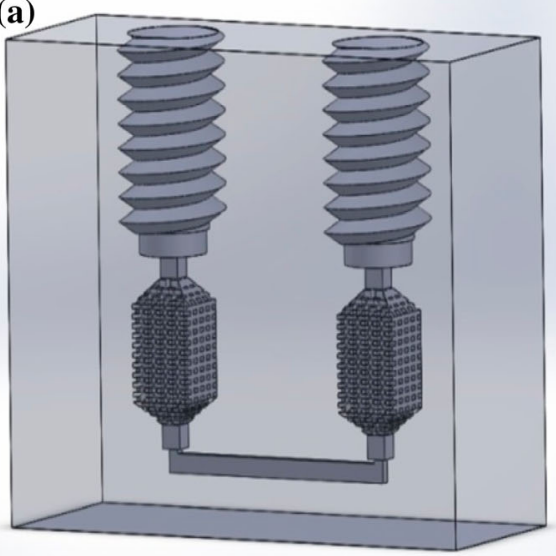

(b)

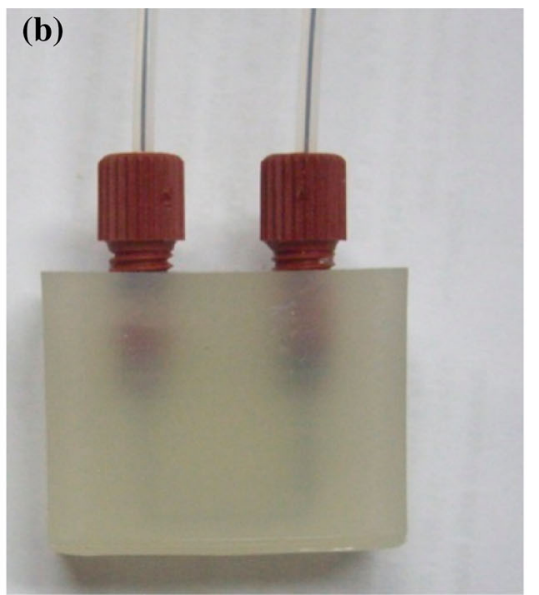

(c)

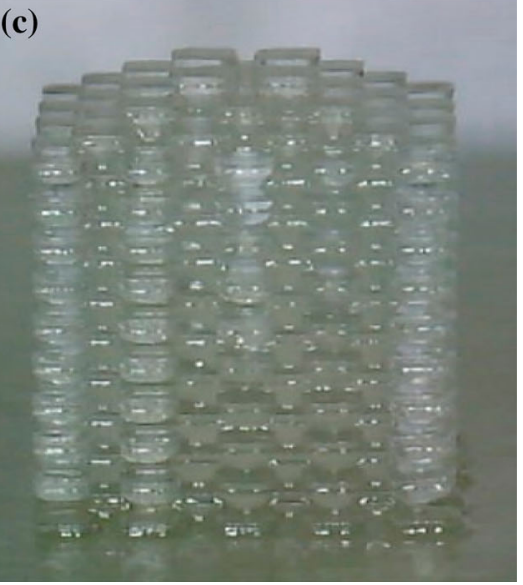

Figure 17 Pictures of the (a) CAD design of the device, (b) 3-D-printed device, (c) 3-D-printed extraction cuboid. Adapted with permission from reference [67]. Copyright [2015], [American Chemical Society].

advantages such as complete isolation and recovery of the catalyst from the reaction mixture [76]. A typical example is a monolithic block [77]. A monolithic block is a system consisting of several microchannels in a regular $3-D$ form. They are gradually replacing traditional powdered catalyst material by helping to overcome their drawbacks. Two major strategies have been reported for the development of structured catalysts via 3-D printing technology. This includes direct printing of the catalytic structure also known as robocasting [78] and the use of a mould, commonly referred to as the templating method [79].

Many studies reporting these catalytic systems have been published; Tubío et al. [80] reported the direct fabrication of a porous $\mathrm{Cu} / \mathrm{Al}_{2} \mathrm{O}_{3}$ woodpile catalytic structure using the robocasting method (Fig. 21a). Similarly, Díaz-Marta et al. [81] reported the same strategy for the preparation of a palladium/copper-supported silica hybrid monolith catalyst (Fig. 21b). In another study, Michorczyk et al. [82] prepared a monolith catalyst using a 3-D-printed template (mould). The monolithic block consists of $\alpha$ - 

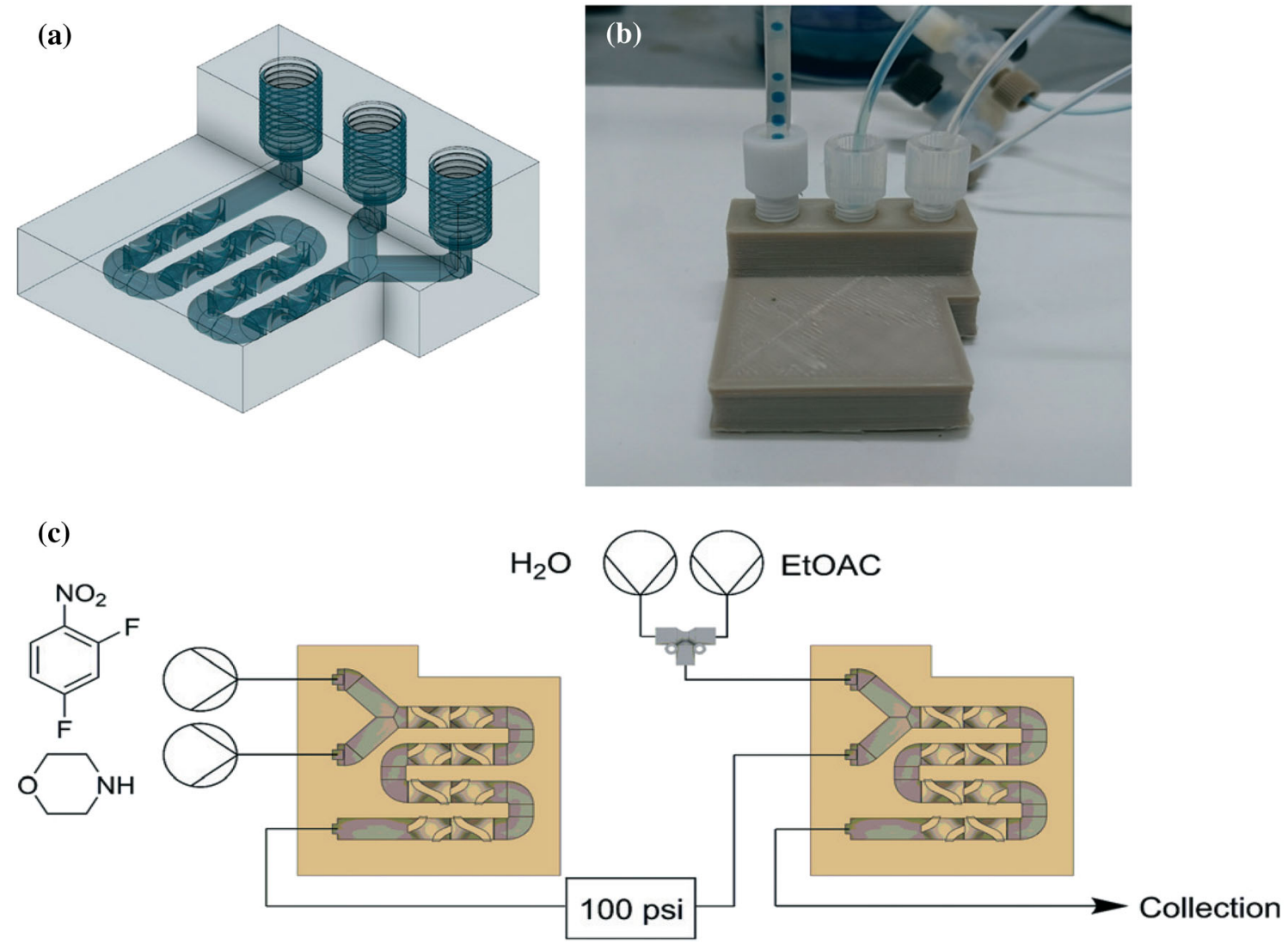

Figure 18 a CAD design of the flow reactor, $\mathbf{b} 3$ - $D$-printed PEEK flow reactor, $\mathbf{c}$ the reaction set-up. Adapted with permission from reference [48]. Copyright [2020], [Royal Society of Chemistry].
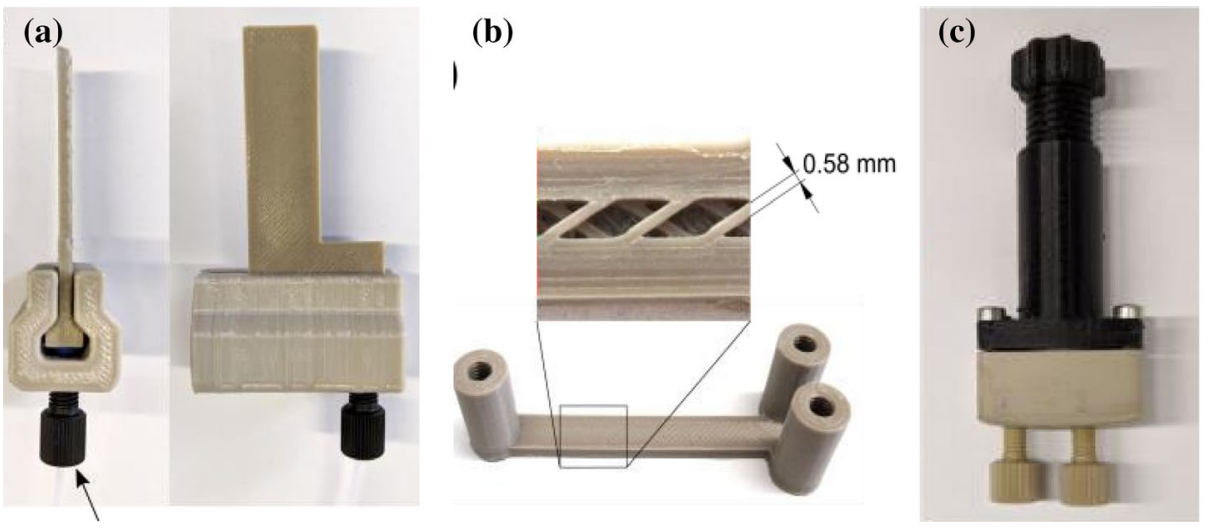

(d)

Figure 19 Images of the 3-D-printed a reactor, $\mathbf{b}$ mixer, $\mathbf{c}$ backpressure regulator, $\mathbf{d}$ separator. Adapted with permission from reference [72]. Copyright [2020], [Royal Society of Chemistry].

$\mathrm{Al}_{2} \mathrm{O}_{3}$ prior to the immobilisation of the catalytically active material (Mn- and $\mathrm{Na}_{2} \mathrm{WO}^{4-}$ ) (Fig. 21c). Similarly, Li et al. also designed and fabricated monoliths of different channel configurations using the $3-D$ printed template method [83] (Fig. 21d). The different channel configuration was used to vary the effect of the flow path within the prepared monolith catalyst.
The monolithic catalyst consists of phenolformaldehyde/aluminium isopropoxide formulation (Fig. 21d).

In all the experiments conducted by references [80-83] using 3-D printing technology for catalyst preparation, they reported impressive activity, stability, and recyclability. Motivated by these results,

\section{Springer}




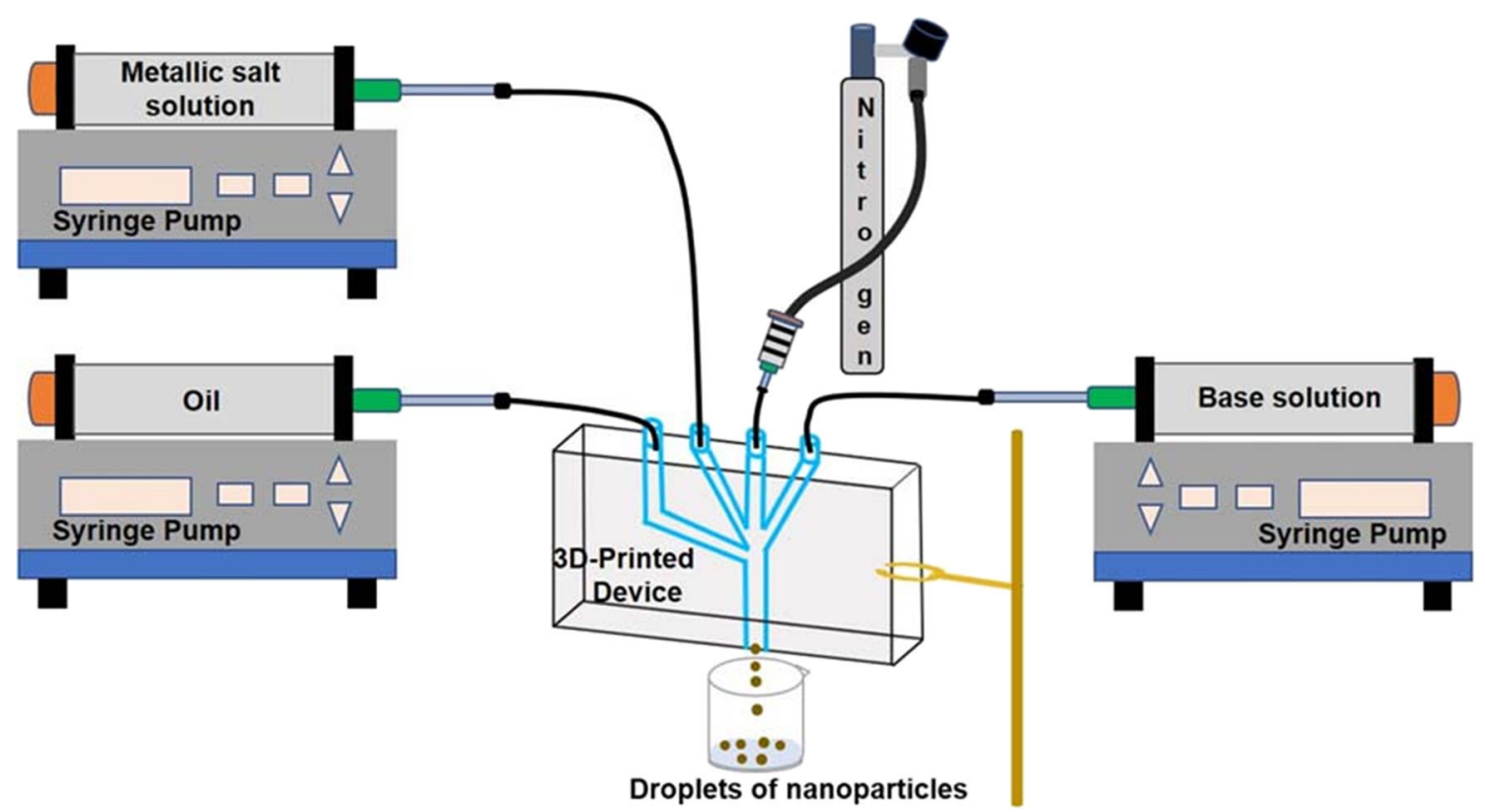

Figure 20 The schematic of the nanoparticle synthesis using the 3-D-printed device. Adapted with permission from reference [73]. Copyright [2020], [Springer].

we have also fabricated palladium-supported monolithic structures using the templating strategy. The palladated monolith was employed for the MizorokiHeck, Suzuki-Miyaura, and copper-free Sonogashira-Hagihara cross-coupling reactions [84]. The picture of the functionalised monolith structure is depicted in Fig. 21e.

A variety of porous catalytic structures have been printed directly or indirectly, such as activated carbon [85], ceramics [86], polymers [87], zeolites [88], and metals and metal oxides [89]. More recently, metal-organic frameworks (MOFs) have continued to gain attention, especially in heterogeneous catalysis. The unique property of MOFs is the extremely high surface area [90] and porosity compared to other porous materials [91]. This makes them an attractive choice in heterogeneous catalysis [92]. A major challenge observed with the use of MOFs is its poor stability and separation problems arising from its crystalline nature, which often limits its catalytic application, especially on a large scale [93]. To address these overriding challenges, MOFs are often pelletised or suspended in polymer matrixes before shaping into functional structures.

However, most traditional techniques of converting crystalline MOFs into pellets and 3-D-structured catalysts often compromised the surface properties and blocked the catalytically active sites [94]. Also, these techniques are not flexible, thus limiting the shape, size, and architecture of the resulting catalyst. Interestingly, 3- $D$ printing has been identified as an attractive choice for the densification of materials because it is flexible, convenient, low cost, and versatile and allows the construction of objects with complex parts [95].

A direct 3-D printing of MOFs was described in the work of Lim et al. [96]. In their study, a self-standing MOFs monolith was printed via a direct ink writing (extrusion-based technique) with minimal additive loading to preserve the functionality of the shaped MOFs monoliths. Also, the printed MOFs demonstrated a very high surface area of $794 \mathrm{~m}^{2} / \mathrm{g}$. The monolithed MOFs, denoted as 3DP-HKUST- $1_{\text {gel }}$, were used for the adsorption of methane. They reported that the efficiency of the printed MOFs was not only comparable to other monoliths shaped by other methods but is also superior. The pictures of the printed monoliths and the performance are presented in Fig. 22a, b, respectively.

In 2020, Liu et al. [97] also reported the successful fabrication of a MOFs hierarchical porous catalyst using a direct ink writing (DIW) method. The procedure was similar to the previous report by Lim et al. [96]. However, the major difference is that the MOF active catalytic material was immobilised on the 3-D-printed ceramic support by wash coating 


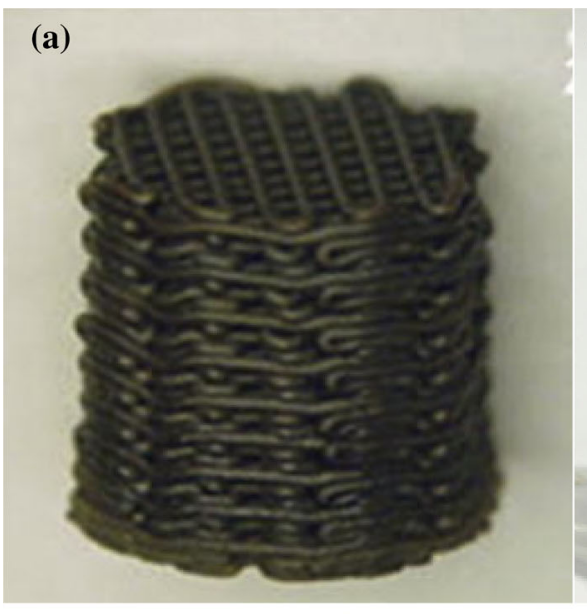

(d)

(I)
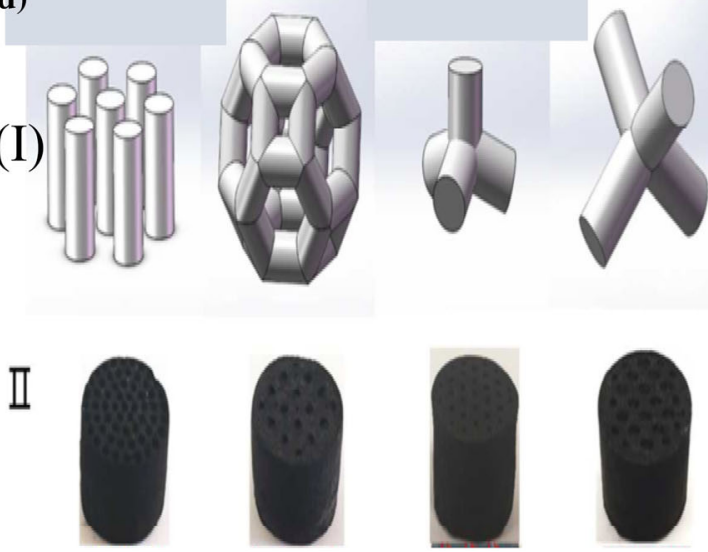

(III)

Figure 21 Pictures of 3-D-printed catalytic material. a 3-Dprinted monolith prepared by direct printing of $\mathrm{Cu} / \mathrm{Al}_{2} \mathrm{O}_{3}$ ink. Adapted with permission from reference [80]. Copyright [2016], [Elsevier]. b 3-D-printed $\mathrm{Pd} / \mathrm{Cu}-\mathrm{SiO}_{2}$ hybrid monolith prepared by robocasting. Adapted with permission from reference [81]. Copyright [2018], [American Chemical Society]. c 3-D-printed monolith prepared by the templating method. Adapted with

(Fig. 23). The printed material (3DP-HPC@HKUST-1) was tested on the degradation of various organic dyes such as methylene blue, rhodamine B, direct red, and malachite green.

As reported in their study, the prepared catalytic structures demonstrated outstanding catalytic performance in all the reactions tested. The catalyst also showed long-time reusability of more than 50 times without a significant loss in catalytic activity.

Lahtinen et al. [93] also reported a successful shaping of copper (II) benzene-1,3,5-tricarboxylate (HKUST) MOFs by 3-D printing. The printable material was prepared by suspending the MOFs in a nylon-12 polymeric material. Unlike the direct extrusion printing technique employed by Lim et al. (c)
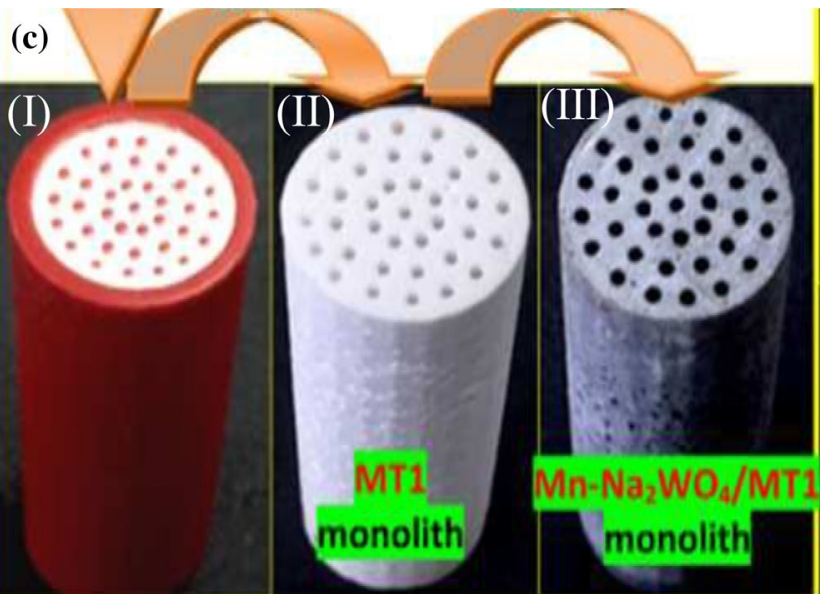

(e) (I)
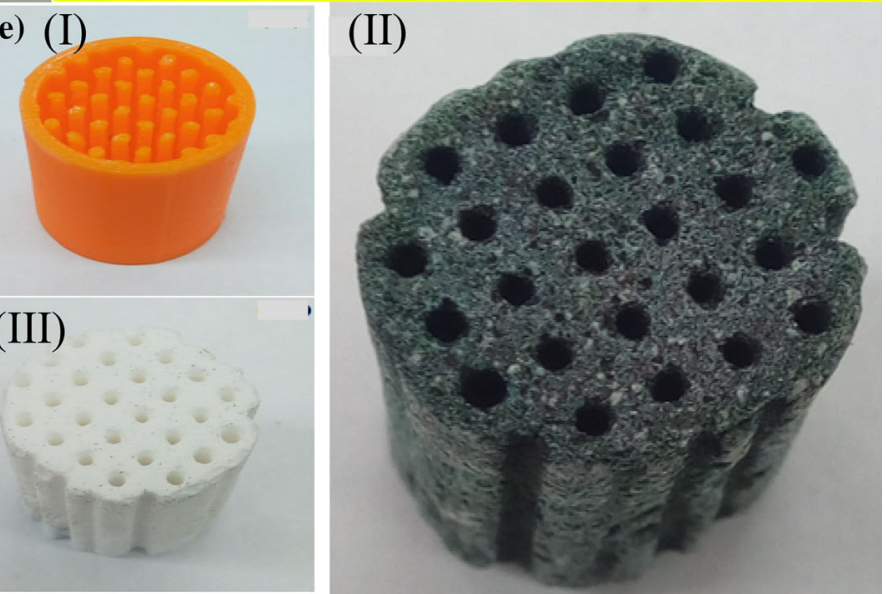

permission from reference [82]. Copyright [2016], [Royal Society of Chemistry]. d 3-D-printed monolith catalyst prepared by the templating method. Adapted with permission from reference [83]. Copyright [2018], [Royal Society of Chemistry]. e 3-D-printed monolith prepared by the templating method. Adapted with permission from reference [64]. Copyright [2020], [Elsevier].

[96], they used the selective laser printing technique 3- $D$ printing process. The image of the printed MOFs is presented in Fig. 24a. The printed catalytic material was utilised for the adsorption of carbon (iv) oxide. The result demonstrates a very good activity coupled with the impressive stability of the fabricated MOFs developed by $3-D$ printing.

Several reports on functionalised 3-D-printed catalytic structures are developed by dispersing, loading, or impregnation of active catalytic material on a polymeric support or carrier [64, 83, 99, 99-102]. The support or the carrier may interfere with the intrinsic property of catalytically active ingredients or vice versa. Therefore, there is an increasing demand for the preparation of stand-alone 3-D-printed catalytic 
(a)

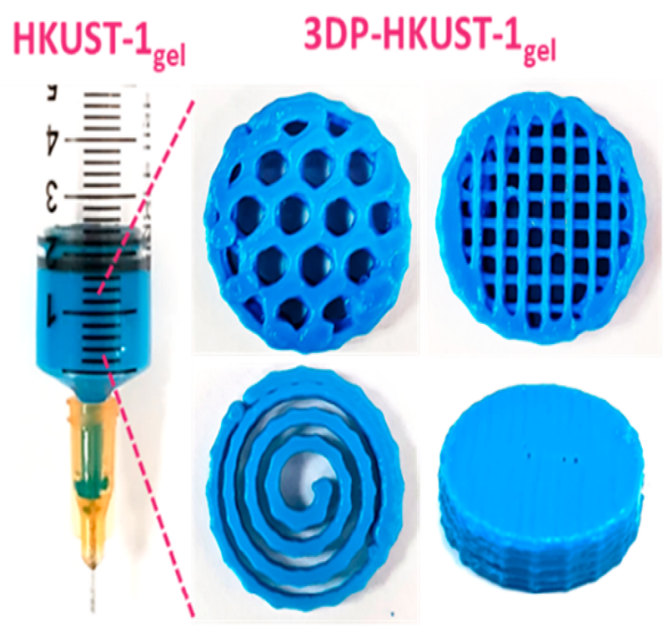

(b)

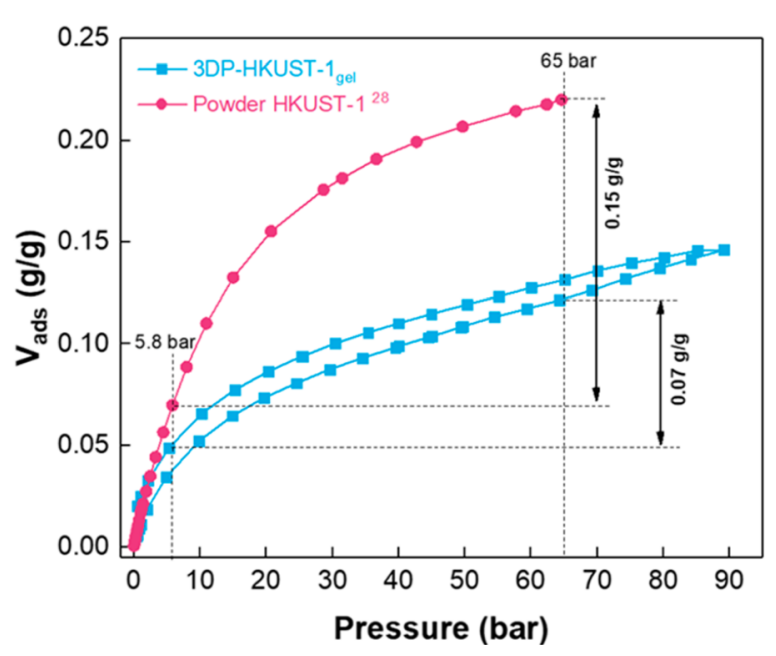

monolith. Adapted with permission from reference [96]. Copyright [2019], [American Chemical Society].
Figure 22 a Picture of the MOF gel (HKUST-1 gel ) and the $3-D$ printed MOF monoliths (3DP-HKUST-1 $\left.1_{\text {gel }}\right)$. b Plot of the methane uptake comparison between powdered MOFs and printed MOFs

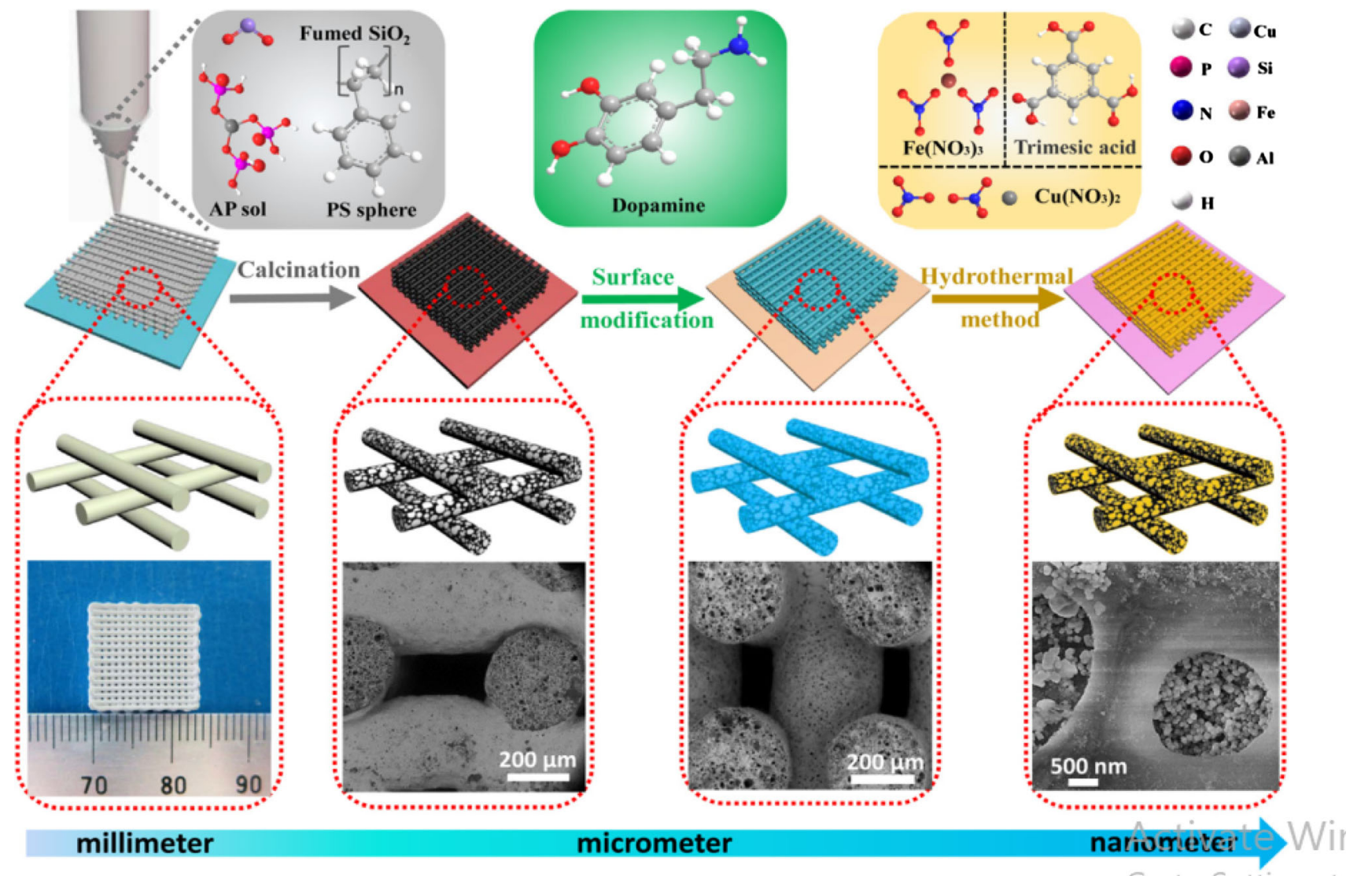

Figure 23 Preparation scheme of the 3-D-printed ceramic support and the MOF surface modification. Adapted with permission from reference [97]. Copyright [2020], [Elsevier].

structures without interfering support, carrier, or binder. Recently, Halevi et al. prepared a 3-D-printed catalytic material consisting of $100 \%$ pure, self-standing nickel coordination polymer [98] using a 

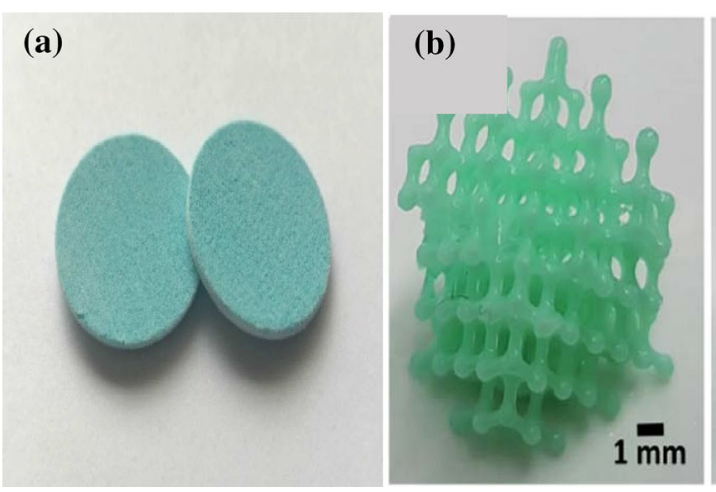

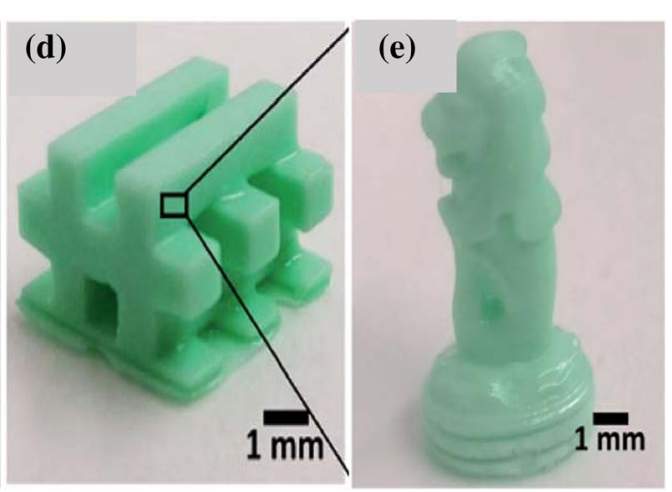

(e)

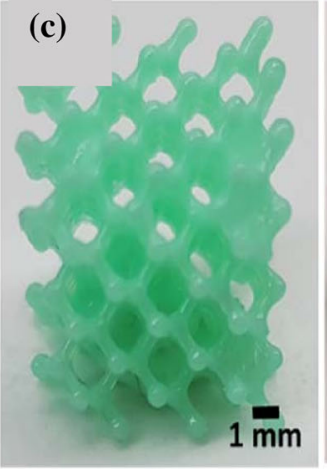

Figure 24 a Picture of the printed MOFs/nylon-12 catalytic disks. Adapted with permission from reference [93]. Copyright [2019], [Wiley]. b, c, d, e Pictures of different architectures of the 3-D-

modified digital light processing printer (DLP). The pictures of the printed MOFs are depicted in Fig. 24b-e.

The scope of 3-D printing in catalysis is not only limited to emerging catalytic applications but has been used to effectively upgrade the existing procedures in the batch process. This was demonstrated in a recent study conducted by Penny et al. [103], and they reported the novel design and fabrication of a catalytically active stirrer bead. As proof of concept, a catalytic material, $p$-toluenesulfonic $(p \mathrm{TsOH})$ acid was prepared to encapsulate the commercial stirrer beads via stereolithographic (SLA) 3-D printing. The stirrer bead was employed in the Mannich addition reaction with acetone, aniline, and benzaldehyde to form a corresponding Mannich base. The same reaction was performed with a blank (standard) stirrer bead as a control experiment, and the activity was compared. The design of the catalytically active printed polyNiComplex catalytic material by Oded et al. Adapted with permission from reference [98]. Copyright [2020], [Royal Society of Chemistry].

stirrer is presented in Fig. 25a, b. The control experiment using $10 \%$ free $p$-toluenesulfonic $(p \mathrm{TsOH})$ gives $88 \%$ yield of the target product, while the $p$ toluenesulfonic ( $p \mathrm{TsOH}$ )-impregnated catalytically active stirrer gives $91 \%$ yield. In addition, the catalytically active stirrer demonstrated a high degree of reusability and operational simplicity.

Bulatov et al. [104] also prepared a catalytic active stirrer bar cover (Fig. 25 c). The printed material consists of a mixture of porous $\mathrm{Pd} / \mathrm{SiO}_{2}$ catalyst and commercially available polypropylene powder as the support. The selective laser 3- $D$ printing technology was employed in the printing process because it offers greater accuracy, reproducibility, and higher resolution than the most commonly used fused deposition modelling technique. The printed catalytically active stirrer was evaluated in batch mode for the hydrogenation of unsaturated hydrocarbon, namely styrene, phenylacetylene, and cyclohexene.

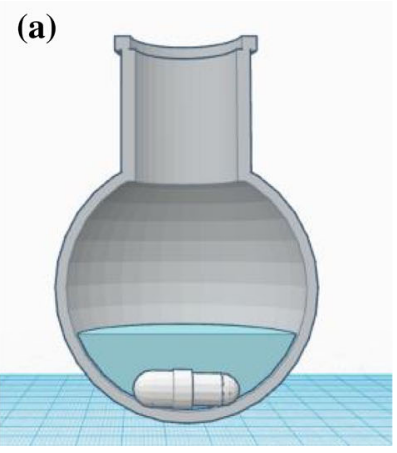

(b)
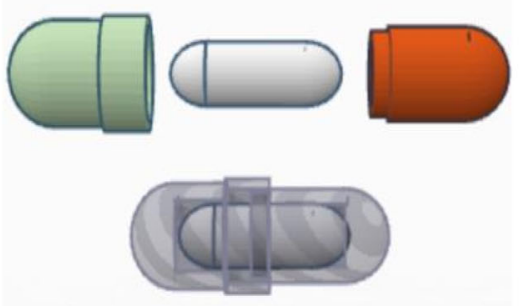

Figure 25 a Picture of a traditional stirrer in a batch reactor. Adapted with permission from reference [103]. Copyright [2020], [Royal Society of Chemistry]. b Picture of $p$-toluenesulfonic $(p \mathrm{TsOH})$ acid-impregnated catalytically active stirrer. Adapted
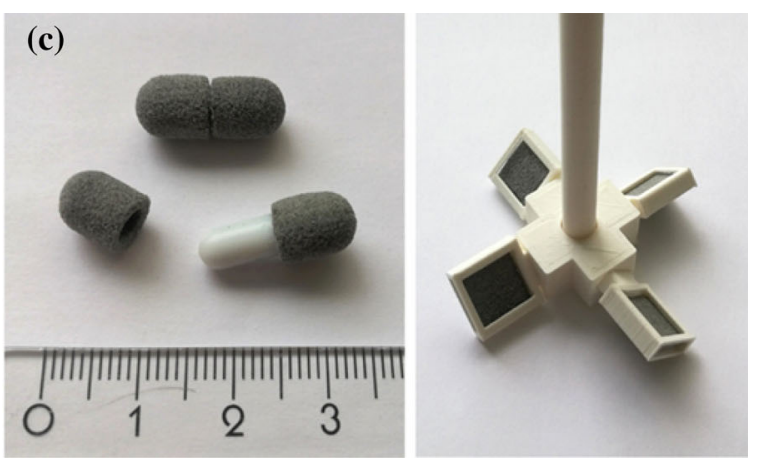

with permission from reference [103]. Copyright [2020], [Royal Society of Chemistry]. c Picture of the catalytically active stir bars. Adapted with permission from reference [104]. Copyright [2020], [Wiley]. 
The results show that the 3-D-printed stirrer was able to successfully catalyse the chosen substrates. Their findings also indicated that the results compare favourably well with the unprinted version of the pure catalyst $\left(\mathrm{Pd} / \mathrm{SiO}_{2}\right)$ powder without any support or carrier. This clearly demonstrates the feasibility of the technique. In addition, the printed catalytically active material was very stable, abrasion-free, and thermally stable, as evidenced by the recyclability results. To further demonstrate their approach's versatility, the authors also performed SuzukiMiyaura cross-coupling with the 3-D-printed stirrer [104], and the result is comparable to the unprinted powdered version of the printed catalyst. They also reported minimal leaching and a convenient recoverability of the catalytically active stirrer bar throughout the experiment.

In another study conducted by Lederle et al. [105], they reported an effective procedure of carrying out inert gas chemistry using 3-D-printed reaction vessels with integrated sample holders for spectroscopic analysis. This included a sealed reaction container with integrated cuvette or NMR tubes to facilitate the reaction, synthesis, and analysis of the product in an inert environment. The reaction vessels are fabricated with a cheap and efficient ABS thermoplastic filament. The pictures of the gas-tight reaction vessels are presented in Fig. 26. The fabricated reaction devices were subjected to a wide range of air-sensitive reactions, including the reduction of trimethylaluminium.
The results obtained from the 3-D-printed reaction vessels demonstrate the practicability of their method. To further expand the application of the fabricated reaction vessels, the Hübner group recently performed the synthesis of aryl naphthyl alkynes via a palladium-catalysed Sonogashira crosscoupling reaction of aryl halides and arylpropiolic acids (Scheme 6) in a 3-D-printed inert gas flask/ spinner combined with NMR tube (Fig. 26h) [106]. The reaction flask was printed with a polyamide thermoplastic filament to promote optically transparent, pressure, and temperature resistance reaction vessels.

While $3-D$ printing is becoming prevalent in modern chemistry laboratories, the digital design of these reactive structures requires a good understanding of computer-aided design (CAD) skills which is not familiar to most synthetic chemists. To overcome this barrier, Hou et al. [107] developed software to design reaction devices with minimal CAD experience. The software is named ChemSCAD (Chemical Synthesis by Computer-Aided Design). It uses a graphical user interface to model reaction devices using only reaction parameters relevant to the synthetic procedure. The digitally generated reaction system was used for the synthesis of ribavirin, modafinil, and lomustine. They reported an impressive performance in all the reactions tested with a $43 \%$ yield in ribavirin, $60 \%$ yield in modafinil, and $65 \%$ yield in lomustine.

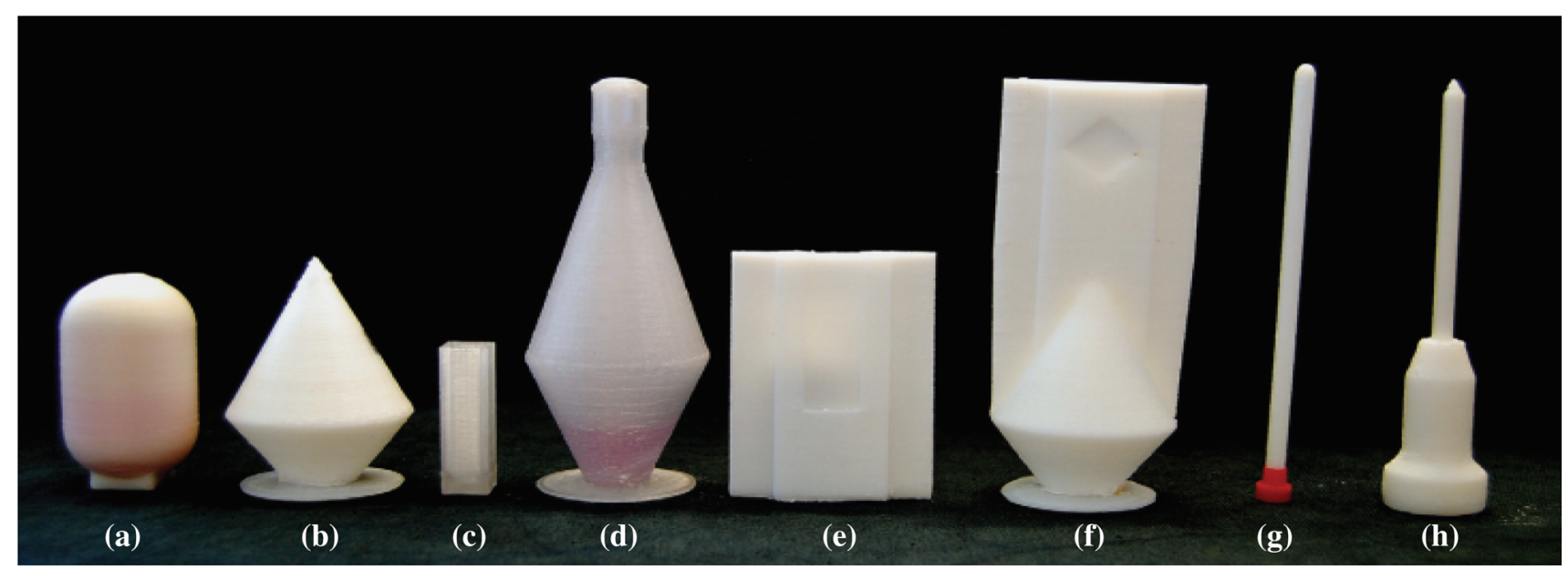

Figure 26 3- $D$-Printed reaction vessels. a Round-bottom flask, b conical flask, c UV cuvette, $\mathbf{d}$ airtight gas flask/UV cuvette, e IR cuvette, $\mathbf{f}$ gas flask/IR cuvette, $\mathbf{g}$ NMR tube, $\mathbf{h}$ inert gas flask/ spinner with NMR tube. Adapted with permission from reference [105]. Copyright [2016], [Wiley]. 


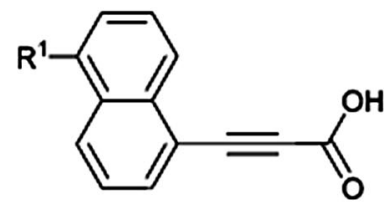

$\mathrm{R}^{1}=\mathrm{H}(1), \mathrm{NO}_{2}(2)$<smiles>[R]c1c2ccccc2c(Br)c2ccccc12</smiles>

$\mathrm{R}^{2}=\mathrm{H}, \mathrm{OMe}, \mathrm{NMe}_{2}$

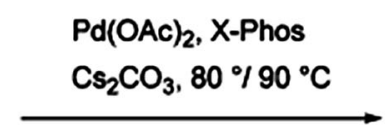

$\left[D_{8}\right]$ THF, $N_{2}$

in 3D-printed tube

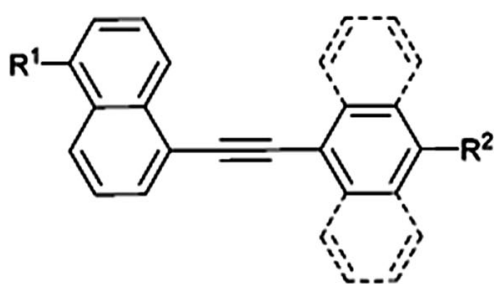

Scheme 6 Sonogashira coupling in 3-D-printed NMR tube. Adapted with permission from reference [106]. Copyright [2017], [Royal Society of Chemistry].

\section{Conclusions}

Going by these literature findings, we conclude that the application of 3-D printing technology in modern-day chemistry is vast. It is not only limited to digital manufacturing of laboratory hardware, analytical instruments, teaching aids, and reactor development, but it is a novel tool for catalyst design and development, robotics, and automation for digital synthesis in chemistry. While the technology has continued to witness exponential growth, it will be uncritical to overlook some challenges currently facing this technique.

A serious challenge with $3-D$ printing is the environmental impact. The waste plastic, resin, and other by-products of additive manufacturing end up in landfills, contaminating the environment. This practice contradicts the current movement of promoting a green environment. Another problem is limited printing materials available for devices fabrication. In addition, the current knowledge gap also translates into a lack of enough personnel with the required skills to design 3-D files and operate a $3-D$ printer.

Despite these challenges, the future of $3-D$ printing is exceedingly bright. As it continues to evolve, it will continue to overcome its limitations and challenges. Factories will not be abandoned, but additive manufacturing techniques will transform the manufacturing process. It will influence the supply chain by decentralising the production of goods and customisation. The majority of the literature reports cited in this review were published in the last four years. This clearly shows that this technology is gaining tremendous attention in chemistry and associated fields.

\section{Acknowledgements}

We gratefully acknowledge the funding supported by the National Research Foundation of South Africa \{Grant specific unique reference number (UID) 5386\}. We acknowledge the University of Johannesburg for the assistance with the TEM analysis in the spectra laboratory. We also acknowledge the support from Mr. D. Harris and Dr. R. Meyer from Shimadzu South Africa for the use of their instrument.

\section{References}

[1] Alvarez PJJ, Chan CK, Elimelech M, Halas NJ, Villagrán D (2018) Emerging opportunities for nanotechnology to enhance water security. Nat Nanotechnol 13:634-641

[2] Tijing LD, Dizon JRC, Ibrahim I, Nisay ARN, Shon HK, Advincula RC (2020) 3D printing for membrane separation, desalination and water treatment. Appl Mater Today $18: 100486$

[3] Zhang Y, Sun X, Nomura N, Fujita T (2019) Hierarchical nanoporous copper architectures via 3D printing technique for highly efficient catalysts. Small 15:1805432

[4] Macdonald NP, Currivan SA, Tedone L, Paull B (2017) Direct production of microstructured surfaces for planar chromatography using 3D printing. Anal Chem 89:2457-2463

[5] Zhang Y, Ge S, Yu J (2016) Trends in Analytical Chemistry Chemical and biochemical analysis on lab-on-a-chip devices fabricated using three-dimensional printing. Trends Anal Chem 85:166-180

[6] Santana HS, Rodrigues AC, Lopes MGM, Russo FN, Silva JL Jr, Taranto OP (2020) 3D printed millireactors for process intensification. Chinese J Chem Eng 28:180-190

[7] Salentijn GIJ, Oomen PE, Grajewski M, Verpoorte E (2017) Fused deposition modeling 3D printing for (bio) analytical device fabrication: procedures, materials, and applications. Anal Chem 89:7053-7061 
[8] Meng C, Ho B, Ng SH, Ho K, Li H, Yoon Y (2015) Lab Chip 15:3627-3637

[9] Anderson KB, Lockwood SY, Martin RS, Spence DM (2013) A 3D printed fluidic device that enables integrated features. Anal Chem 85:5622-5626

[10] Rossi S, Porta R, Brenna D, Puglisi A, Benaglia M (2017) Stereoselective catalytic synthesis of active pharmaceutical ingredients in homemade 3D-printed mesoreactors. Angew Chemie 129:4354-4358

[11] Liu C, Huang N, Xu F, Tong J, Chen Z, Gui X, Fu Y, Lao C (2018) 3D printing technologies for flexible tactile sensors toward wearable electronics and electronic skin. Polymers (Basel) 10:629

[12] Melnikova R, Ehrmann A, Finsterbusch K (2014) 3D printing of textile-based structures by fused deposition modelling (FDM) with different polymer materials. In: IOP conference series: materials science and engineering. IOP publishing, p 12018

[13] Duarte LC, Chagas CLS, Ribeiro LEB, Coltro WKT (2017) Sensors and Actuators B: chemical 3D printing of microfluidic devices with embedded sensing electrodes for generating and measuring the size of microdroplets based on contactless conductivity detection. Sensors Actuators B Chem 251:427-432

[14] Kadimisetty K, Mosa IM, Malla S, Satterwhite-Warden JE, Kuhns TM, Faria RC, Lee NH, Rusling JF (2016) 3Dprinted supercapacitor-powered electrochemiluminescent protein immunoarray. Biosens Bioelectron 77:188-193

[15] Kitson PJ, Symes MD, Dragone V, Cronin L (2013) Combining 3D printing and liquid handling to produce user-friendly reactionware for chemical synthesis and purification. Chem Sci 4:3099-3103

[16] Sharma M, Reddy SV, Kulkarni AA (2014) 3D flow reactors: flow, hydrodynamics, and performance. Ind Eng Chem Res 53:1916-1923

[17] Saggiomo V (2020) 3D Printed devices for catalytic systems. Wiley, Catal immobil methods appl, pp 369-408

[18] Ambrosi A, Pumera M (2016) 3D-printing technologies for electrochemical applications. Chem Soc Rev 45:2740-2755

[19] Lee W, Kwon D, Choi W, Jung GY, Au AK, Folch A, Jeon $\mathrm{S}$ (2015) 3D-printed microfluidic device for the detection of pathogenic bacteria using size-based separation in helical channel with trapezoid cross-section. Sci Rep 5:7717

[20] Wei X, Li D, Jiang W, Gu Z, Wang X, Zhang Z, Sun Z (2015) 3D printable graphene composite. Sci Rep 5:11181

[21] Wang Z, Wang J, Li M, Sun K, Liu C (2014) Three-dimensional printed acrylonitrile butadiene styrene framework coated with $\mathrm{Cu}$-BTC metal-organic frameworks for the removal of methylene blue. Sci Rep 4:5939
[22] Kitson PJ, Glatzel S, Chen W, Lin CG, Song YF, Cronin L (2016) 3D printing of versatile reactionware for chemical synthesis. Nat Protoc 11:920-936

[23] Hussain SI. (2019) 3D printing has emerged to be one of the biggest inventions ever. Linkedin blog. https://www. linkedin.com/pulse/3d-printing-has-emerged-one-biggestin ventions-ever-shehroz-hussain/. Accessed 10 Aug 2021

[24] Gibson I, Rosen DW, Stucker B (2014) Additive manufacturing technologies. Springer

[25] Baumann FW (2018) Thirty thousand 3D models from thingiverse. Data 3:5

[26] Capel AJ, Rimington RP, Lewis MP, Christie SDR (2018) $3 \mathrm{D}$ printing for chemical, pharmaceutical and biological applications. Nat Rev Chem 2:422-436

[27] Sule SS, Petsiuk AL, Pearce JM (2019) Open source completely 3 -D printable centrifuge. Instruments 3:30

[28] Dhankani KC, Pearce JM (2017) Open source laboratory sample rotator mixer and shaker. HardwareX 1:1-12

[29] Trivedi DK, Pearce JM (2017) Open source 3-D printed nutating mixer. Appl Sci 7:942

[30] Cecil F, Zhang M, Guijt RM, Henderson A, Nesterenko PN, Paull B, Breadmore MC, Macka M (2017) 3D printed LED based on-capillary detector housing with integrated slit. Anal Chim Acta 965:131-136

[31] Whitehead HD, Waldman JV, Wirth DM, LeBlanc G (2017) $3 \mathrm{D}$ printed UV-visible cuvette adapter for low-cost and versatile spectroscopic experiments. ACS Omega 2:6118-6122

[32] Scalfani VF, Vaid TP (2014) 3D printed molecules and extended solid models for teaching symmetry and point groups. J Chem Educ 91:1174-1180

[33] Penny MR, Cao ZJ, Patel B, Sil dos Santos B, Asquith CRM, Szulc BR, Rao ZX, Muwaffak Z, Malkinson JP, Hilton ST (2017) Three-dimensional printing of a scalable molecular model and orbital kit for organic chemistry teaching and learning. J Chem Educ 94:1265-1271

[34] Jones OAH, Spencer MJS (2018) A simplified method for the $3 \mathrm{D}$ printing of molecular models for chemical education. J Chem Educ 95:88-96

[35] Rossi S, Benaglia M, Brenna D, Porta R, Orlandi M (2015) Three dimensional (3D) printing: a straightforward, userfriendly protocol to convert virtual chemical models to reallife objects. J Chem Educ 8:1398-1401

[36] Stone-Sundberg J, Kaminsky W, Snyder T, Moeck P (2015) $3 \mathrm{D}$ printed models of small and large molecules, structures and morphologies of crystals, as well as their anisotropic physical properties. Cryst Res Technol 50:432-441

[37] Wood PA, Sarjeant AA, Bruno IJ, Macrae CF, MaynardCasely HE, Towler M (2017) The next dimension of structural science communication: simple 3D printing 
directly from a crystal structure. CrystEngComm 19:690-698

[38] Robertson MJ, Jorgensen WL (2015) Illustrating concepts in physical organic chemistry with $3 \mathrm{D}$ printed orbitals. J Chem Educ 92:2113-2116

[39] Casas L, Estop E (2015) Virtual and printed 3D models for teaching crystal symmetry and point groups. J Chem Educ 92:1338-1343

[40] He Y, Wu Y, Fu J, Gao Q, Qiu J (2016) Developments of 3D printing microfluidics and applications in chemistry and biology: a review. Electroanalysis 28:1658-1678

[41] Wei Q, Li H, Liu G, He Y, Wang Y, Tan YE, Wang D, Peng X, Yang G, Tsubaki N (2020) Metal 3D printing technology for functional integration of catalytic system. Nat Commun $11: 1-8$

[42] Chorkendorff I, Niemantsverdriet JW (2017) Concepts of modern catalysis and kinetics. Wiley

[43] Tsao C-W (2016) Polymer microfluidics: simple, low-cost fabrication process bridging academic lab research to commercialised production. Micromachines 7:225

[44] Hu X, Karnetzke J, Fassbender M, Drücker S, Bettermann S, Schroeter B, Pauer W, Moritz H-U, Fiedler B, Luinstra G (2020) Smart reactors-Combining stimuli-responsive hydrogels and 3D printing. Chem Eng J 387:123413

[45] Symes MD, Kitson PJ, Yan J, Richmond CJ, Cooper GJT, Bowman RW, Vilbrandt T, Cronin L (2012) Integrated 3Dprinted reactionware for chemical synthesis and analysis. Nat Chem 4:349

[46] Manzano JS, Wang H, Slowing II (2019) High throughput screening of 3D printable resins: adjusting the surface and catalytic properties of multifunctional architectures. ACS Appl Polym Mater 1:2890-2896

[47] Lahtinen E, Turunen L, Hänninen MM, Kolari K, Tuononen HM, Haukka M (2019) Fabrication of porous hydrogenation catalysts by a selective laser sintering $3 \mathrm{D}$ printing technique. ACS Omega 4:12012-12017

[48] Harding MJ, Brady S, O'Connor H, Lopez-Rodriguez R, Edwards MD, Tracy S, Dowling D, Gibson G, Girard KP, Ferguson S (2020) 3D printing of PEEK reactors for flow chemistry and continuous chemical processing. React Chem Eng 5:728-735

[49] Anciaux SK, Geiger M, Bowser MT (2016) 3D printed micro free-flow electrophoresis device. Anal Chem $88: 7675-7682$

[50] Dilberoglu UM, Gharehpapagh B, Yaman U, Dolen M (2017) The role of additive manufacturing in the era of industry 4.0. Procedia Manuf 11:545-554

[51] Kalsoom U, Nesterenko PN, Paull B (2018) Current and future impact of $3 \mathrm{D}$ printing on the separation sciences. TrAC Trends Anal Chem 105:492-502
[52] Dávila JSM (2018) Customizable heterogeneous catalysts: from 3D printed designs to mesoporous materials. Doctoral dissertation, Iowa State University. https://doi.org/10.2172/ 1505174

[53] Rao ZX, Patel B, Monaco A, Cao ZJ, Barniol-Xicota M, Pichon E, Ladlow M, Hilton ST (2017) 3D-printed polypropylene continuous-flow column reactors: exploration of reactor utility in SNAr reactions and the synthesis of bicyclic and tetracyclic heterocycles. European J Org Chem 2017:6499-6504

[54] Neumaier JM, Madani A, Klein T, Ziegler T (2019) Lowbudget 3D-printed equipment for continuous flow reactions. Beilstein J Org Chem 15:558-566

[55] Dragone V, Sans V, Rosnes MH, Kitson PJ, Cronin L (2013) 3D-printed devices for continuous-flow organic chemistry. Beilstein J Org Chem 9:951-959

[56] Capel AJ, Edmondson S, Christie SDR, Goodridge RD, Bibb RJ, Thurstans M (2013) Design and additive manufacture for flow chemistry. Lab Chip 13:4583-4590

[57] Kitson PJ, Marie G, Francoia J-P, Zalesskiy SS, Sigerson RC, Mathieson JS, Cronin L (2018) Digitisation of multistep organic synthesis in reactionware for on-demand pharmaceuticals. Science 359:314-319

[58] Kitson PJ, Glatzel S, Cronin L (2016) The digital code driven autonomous synthesis of ibuprofen automated in a 3D-printer-based robot. Beilstein J Org Chem $12: 2776-2783$

[59] Gromski PS, Granda JM, Cronin L (2020) Universal chemical synthesis and discovery with 'the chemputer.' Trends Chem 1:4-12

[60] Nathan S (2018) "Chemputer" may democratise pharmaceutical sector. The engineer. https://www.theengineer.co. uk/chemputer-synthesis-pharmaceuticals/. Accessed 10 Aug 2021

[61] Angelone D, Hammer AJS, Rohrbach S, Krambeck S, Granda JM, Wolf J, Zalesskiy S, Chisholm G, Cronin L (2021) Convergence of multiple synthetic paradigms in a universally programmable chemical synthesis machine. Nat Chem 13:63-69

[62] Alimi OA, Bingwa N, Meijboom R (2019) Homemade 3-D printed flow reactors for heterogeneous catalysis. Chem Eng Res Des 150:116-129

[63] Alimi OA, Akinnawo CA, Onisuru OR, Meijboom R (2020) 3-D printed microreactor for continuous flow oxidation of a flavonoid. J Flow Chem 10:517-531

[64] Alimi OA, Ncongwane TB, Meijboom R (2020) Design and fabrication of a monolith catalyst for continuous flow epoxidation of styrene in polypropylene printed flow reactor. Chem Eng Res Des 159:395-409 
[65] Au AK, Lee W, Folch A (2014) Mail-order microfluidics: evaluation of stereolithography for the production of microfluidic devices. Lab Chip 14:1294-1301

[66] Zheng Q, Aiello A, Choi YS, Tarr K, Shen H, Durkin DP, Shuai D (2020) 3D Printed photoreactor with immobilized graphitic carbon nitride: a sustainable platform for solar water purification. J Hazard Mater 399:123097

[67] Su C-K, Peng P-J, Sun Y-C (2015) Fully 3D-printed preconcentrator for selective extraction of trace elements in seawater. Anal Chem 87:6945-6950

[68] Chaparro-Garnica CY, Jordá-Faus P, Bailón-García E, Ocampo-Pérez R, Aguilar-Madera CG, Davó-Quiñonero A, Lozano-Castelló D, Bueno-López A (2020) Customizable heterogeneous catalysts: nonchanneled advanced monolithic supports manufactured by 3D-Printing for improved active phase coating performance. ACS Appl Mater Interfaces 12:54573-54584

[69] Garcia-Gonzalez D, Rusinek A, Jankowiak T, Arias A (2015) Mechanical impact behavior of polyether-etherketone (PEEK). Compos Struct 124:88-99

[70] Valentan B, Kadivnik Ž, Brajlih T, Anderson A, Drstvenšek I (2013) Processing poly (ether etherketone) an a 3D printer for thermoplastic modelling. Mater Tehnol 47:715-721

[71] Yang C, Tian X, Li D, Cao Y, Zhao F, Shi C (2017) Influence of thermal processing conditions in $3 \mathrm{D}$ printing on the crystallinity and mechanical properties of PEEK material. J Mater Process Technol 248:1-7

[72] Menzel F, Klein T, Ziegler T, Neumaier J (2020) 3D-printed PEEK reactors and development of a complete continuous flow system for chemical synthesis. React Chem Eng $5: 1300-1310$

[73] Singh V (2020) 3D-printed device for synthesis of magnetic and metallic nanoparticles. J Flow Chem 1:1-8

[74] Soliman A, AlAmoodi N, Karanikolos GN, Doumanidis CC, Polychronopoulou K (2020) A review on new 3-D printed materials' geometries for catalysis and adsorption: paradigms from reforming reactions and $\mathrm{CO}_{2}$ capture. Nanomaterials 10:2198

[75] Rossi S, Puglisi A, Raimondi LM, Benaglia M (2020) Stereolithography 3D-printed catalytically active devices in organic synthesis. Catalysts 10:109

[76] Benaglia M, Puglisi A, Cozzi F (2003) Polymer-supported organic catalysts. Chem Rev 103:3401-3430

[77] Li X, Su H, Ren G, Wang S (2015) A highly stable Pd/ $\mathrm{SiO}_{2} /$ cordierite monolith catalyst for 2-ethyl-anthraquinone hydrogenation. RSC Adv 5:100968-100977

[78] Miranda P, Pajares A, Saiz E, Tomsia AP, Guiberteau F (2008) Mechanical properties of calcium phosphate scaffolds fabricated by robocasting. J Biomed Mater Res Part A
An Off J Soc Biomater Japanese Soc Biomater Aust Soc Biomater Korean Soc Biomater 85:218-227

[79] Comina G, Suska A, Filippini D (2014) PDMS lab-on-achip fabrication using 3D printed templates. Lab Chip 14:424-430

[80] Tubío CR, Azuaje J, Escalante L, Coelho A, Guitián F, Sotelo E, Gil A (2016) 3D printing of a heterogeneous copper-based catalyst. J Catal 334:110-115

[81] Díaz-Marta AS, Tubío CR, Carbajales C, Fernández C, Escalante L, Sotelo E, Guitián F, Barrio VL, Gil A, Coelho A (2018) Three-dimensional printing in catalysis: combining 3D heterogeneous copper and palladium catalysts for multicatalytic multicomponent reactions. ACS Catal 8:392-404

[82] Michorczyk P, Hędrzak E, Węgrzyniak A (2016) Preparation of monolithic catalysts using $3 \mathrm{D}$ printed templates for oxidative coupling of methane. J Mater Chem A 4:18753-18756

[83] Li Y, Chen S, Cai X, Hong J, Wu X, Xu Y, Zou J, Chen BH (2018) Rational design and preparation of hierarchical monoliths through 3D printing for syngas methanation. J Mater Chem A 6:5695-5702

[84] Alimi OA, Akinnawo CA, Meijboom R (2020) Monolith catalyst design via 3D printing: a reusable support for modern palladium-catalysed cross-coupling reactions. New J Chem 44:18867-18878

[85] Yang Y, Shi X, Zhao M, Chu S, Xiao J (2020) Heterogeneous catalytic ozonation of phenol by a novel binary catalyst of Fe-Ni/MAC. Catalysts 10:1123

[86] Shifu C, Xuqiang L, Yunzhang L, Gengyu C (2007) The preparation of nitrogen-doped $\mathrm{TiO}_{2}-\mathrm{xNx}$ photocatalyst coated on hollow glass microbeads. Appl Surf Sci 253:3077-3082

[87] Ligon SC, Liska R, Stampfl J, Gurr M, Mülhaupt R (2017) Polymers for 3D printing and customised additive manufacturing. Chem Rev 117:10212-10290

[88] Li X, Rezaei F, Rownaghi AA (2018) 3D-printed zeolite monoliths with hierarchical porosity for selective methanol to light olefin reaction. React Chem Eng 3:733-746

[89] Abudula T, Qurban RO, Bolarinwa SO, Mirza AA, Pasovic M, Memic A (2020) 3D printing of metal/metal oxide incorporated thermoplastic nanocomposites with antimicrobial properties. Front Bioeng Biotechnol 8:568186

[90] Liu W, Huang J, Yang Q, Wang S, Sun X, Zhang W, Liu J, Huo F (2017) Multi-shelled hollow metal-organic frameworks. Angew Chemie Int Ed 56:5512-5516

[91] Shen K, Zhang L, Chen X, Liu L, Zhang D, Han Y, Chen J, Long J, Luque R, Li Y (2018) Ordered macro-microporous metal-organic framework single crystals. Science 359:206-210 
[92] Wang C, Kim J, Malgras V, Na J, Lin J, You J, Zhang M, Li J, Yamauchi Y (2019) Metal-organic frameworks and their derived materials: emerging catalysts for a sulfate radicalsbased advanced oxidation process in water purification. Small 15:1900744

[93] Lahtinen E, Precker RLM, Lahtinen M, Hey-Hawkins E, Haukka M (2019) Selective laser sintering of metal-organic frameworks: production of highly porous filters by 3D printing onto a polymeric matrix. ChemPlusChem $84: 222-225$

[94] Tian T, Velazquez-Garcia J, Bennett TD, Fairen-Jimenez D (2015) Mechanically and chemically robust ZIF-8 monoliths with high volumetric adsorption capacity. J Mater Chem A 3:2999-3005

[95] Kenel C, Casati NPM, Dunand DC (2019) 3D ink-extrusion additive manufacturing of $\mathrm{CoCrFeNi}$ high-entropy alloy micro-lattices. Nat Commun 10:1-8

[96] Lim GJH, Wu Y, Shah BB, Koh JJ, Liu CK, Zhao D, Cheetham AK, Wang J, Ding J (2019) 3D-Printing of pure metal-organic framework monoliths. ACS Mater Lett $1: 147-153$

[97] Liu D, Jiang P, Li X, Liu J, Zhou L, Wang X, Zhou F (2020) 3D printing of metal-organic frameworks decorated hierarchical porous ceramics for high-efficiency catalytic degradation. Chem Eng J 397:125392

[98] Halevi O, Chen J, Thangavel G, Morris SA, Ben UT, Tischler YR, Lee PS, Magdassi S (2020) Synthesis through 3D printing: formation of $3 \mathrm{D}$ coordination polymers. RSC Adv 10:14812-14817

[99] Nandi M, Uyama H (2014) Porous acrylate monolith supported Pd nanoparticles: highly active and reusable catalyst for Suzuki-Miyaura reaction in water. RSC Adv 4:20847-20855

[100] Quintanilla A, Casas JA, Miranzo P, Osendi MI, Belmonte M (2018) 3D-Printed Fe-doped silicon carbide monolithic catalysts for wet peroxide oxidation processes. Appl Catal B Environ 235:246-255

[101] Kundu D, Patra AK, Sakamoto J, Uyama H (2014) A palladium-loaded mesoporous polymer monolith as reusable heterogeneous catalyst for cross-coupling reactions. React Funct Polym 79:8-13

[102] Bavykina AV, Olivos-Suarez AI, Osadchii D, Valecha R, Franz R, Makkee M, Kapteijn F, Gascon J (2017) Facile method for the preparation of covalent triazine framework coated monoliths as catalyst support: applications in $\mathrm{C} 1$ catalysis. ACS Appl Mater Interfaces 9:26060-26065

[103] Penny MR, Hilton ST (2020) Design and development of 3D printed catalytically-active stirrers for chemical synthesis. React Chem Eng 5:853-858

[104] Bulatov E, Lahtinen E, Kivijärvi L, Hey-Hawkins E, Haukka M (2020) 3D printed palladium catalyst for SuzukiMiyaura cross-coupling reactions. ChemCatChem $12: 4831-4838$

[105] Lederle F, Kaldun C, Namyslo JC, Hübner EG (2016) 3Dprinting inside the glovebox: a versatile tool for inert-gas chemistry combined with spectroscopy. Helv Chim Acta 99:255-266

[106] Lederle F, Meyer F, Kaldun C, Namyslo JC, Hübner EG (2017) Sonogashira coupling in 3D-printed NMR cuvettes: synthesis and properties of arylnaphthylalkynes. New J Chem 41:1925-1932

[107] Hou W, Bubliauskas A, Kitson PJ, Francoia J-P, PowellDavies H, Gutierrez JMP, Frei P, Manzano JS, Cronin L (2021) Automatic generation of 3D-printed reactionware for chemical synthesis digitization using chemSCAD. ACS Cent Sci 7:212-218

Publisher's Note Springer Nature remains neutral with regard to jurisdictional claims in published maps and institutional affiliations. 